\title{
An extension of the notion of the order of a distribution
}

\author{
M. Mišur ${ }^{1,2} \cdot$ L. Palle ${ }^{1,3}$
}

Received: 9 October 2018 / Accepted: 23 March 2019 / Published online: 4 April 2019

(c) Fondazione Annali di Matematica Pura ed Applicata and Springer-Verlag GmbH Germany, part of Springer Nature 2019

\begin{abstract}
In this article we review the notion of the order of a distribution and extend it to the case of positive real numbers. We suggest to use the name Hölder distributions for such distributions. The first part of the paper concerns itself with functional-analytic properties of the Hölder test function spaces and its duals. Of particular interest are the $C_{c}^{r, \alpha+}(\Omega)$ and the $\mathcal{D}_{(r+\alpha)+}^{\prime}(\Omega)$ spaces which have notably better properties such as reflexivity, compared to the classical Hölder spaces. We also give a few examples and some Fourier-analytic properties of distributions of fractional order, and at the end, we note how one can extend classical results where estimates of the order of distributions appear, such as giving a bound on the order of convolution of distributions.
\end{abstract}

Keywords Distributions · Fractional order · Hölder continuous · Fourier transform • Convolution

Mathematics Subject Classification 46F05 (primary), 46A04, 46E15

\section{Introduction}

In his pioneering book on distributions [23], Schwartz introduced the notion of the order of a distribution. A distribution $u \in \mathcal{D}^{\prime}(X)$ is said to be of order smaller or equal to $r$ if the restriction of $u$ to $C_{c}^{\infty}(X ; K)$ is continuous with respect to the topology induced by that of $C_{c}^{r}(X ; K)$, for every compact $K \subset X$. The order of a distribution is the smallest integer $r$ with such a property. A distribution of finite order can be tested against a function that is not infinitely differentiable. In some applications, this means that if our distribution is of finite order, we do not have to consider partial differential equations with only smooth coefficients.

L. Palle

ljpalle@math.hr; palle@math.uni-kiel.de

M. Mišur

mmisur@math.hr; marin.misur@visagetechnologies.com

1 Faculty of Science, University of Zagreb, Bijenička cesta 30, 10000 Zagreb, Croatia

2 Present Address: Visage Technologies AB, Diskettgatan 11A, 58335 Linköping, Sweden

3 Present Address: Christian-Albrechts-Universität zu Kiel, Mathematisches Seminar, Ludewig-Meyn-Str. 6, 24118 Kiel, Germany 
Furthermore, it allows one to obtain results like the structure theorem of distributions where, at least locally, one can represent any distribution as a derivative of some finite order of a continuous function.

This article is motivated by an observation that sometimes the regularity of a test function for a distribution of finite order can be even lower than its order, but not too much (the classical example being the vp. $\left(\frac{1}{x}\right)$ distribution, as discussed in, e.g., Footnote 1 on p. 18 of [24]). We provide a mathematical framework, write down the details to explain this phenomenon, and also provide a few examples and applications, together with a few generalisations of classical results from distribution theory.

The structure of the article is the following. In Sect. 2, we define Hölder test function spaces and examine their properties, and in Sect. 3, we consider their duals which we shall call Hölder distributions. Of particular interest are the spaces $C_{c}^{r, \alpha+}(\Omega)$ and $\mathcal{D}_{(r+\alpha)+}^{\prime}(\Omega)$ since it turns out that they have much better properties. In Sect. 4 , we prove though that they are not nuclear. The following Sect. 5 gives a few examples of distributions and their real order. Finally, we deal with some further properties of Hölder distributions, namely Fourier-analytic properties (Sect. 6), and the order of convolution of Hölder distributions (Sect. 7). At the end of the article, we discuss possible further extensions and related results (Sect. 8).

Let us remark that a special case of Hölder distributions, which we shall introduce in this article, was already considered by Francis Bonahon in $[5,6]$ with applications to the theory of transverse structures for measured laminations on a closed surface of negative Euler characteristic (i.e., a surface of hyperbolic type). We briefly describe the problem which Bonahon resolved by introducing the notion of Hölder distribution. A geodesic lamination on a surface is a lamination whose leaves are geodesic. One can consider several transverse structures for a given geodesic lamination. For example, if one uses Radon measures on arcs transverse to the lamination which satisfy some additional invariance conditions, one constructs classical transverse measures, which have been well studied in the theory of foliations and laminations. But one can consider things more general and use distributions on arcs. An important problem which one must overcome in this consideration is the invariance conditions. Namely, given two homotopic arcs, the homotopy should send the distribution defined on one arc to a distribution defined on the other arc. But this implies that the homotopy between the two arcs must be differentiable, which seldomly happens. In general, a geodesic lamination never admits transverse differentiable structures. But, as Francis Bonahon noticed in [5,6], the homotopy can be chosen to be Hölder, or even Lipschitz, continuous. Once having shown that geodesic laminations have a transverse Hölder structure, he introduced the notion of Hölder distributions as continuous linear functionals on the space of all Hölder functions with compact support. This corresponds to our space $\mathcal{D}_{0+}^{\prime}$ (see Definition 8). For results and applications which stemmed from this, we refer the reader to the survey article [7] and the unfinished monograph [8], which is available online.

At the end of this section, let us give a notation remark. In this article, we use the usual convention for the symbols $\lesssim, \gtrsim$, and $\sim$. For example, $A \lesssim B$ means there is a positive constant $C$ such that $A \leq C B$, and $C$ does not depend on relevant parameters in the given context. $A \lesssim p B$ means there is a constant $C=C(p)$ such that $A \leq C B$, and the constant $C$ depends on the parameter $p$. Sometimes we also use $A \ll B$ (resp. $A \gg B$ ). It means that $A \leq c B$ (resp. $B \leq c A$ ) for some sufficiently small and positive constant $c$. 


\section{Properties of spaces of Hölder continuous test functions}

Let $\Omega \subseteq \mathbf{R}^{d}$ be an open set. For $r \in \mathbf{N}_{0}$ and $\alpha \in\langle 0,1]$, we denote by $C^{r, \alpha}(\Omega)$ the vector space of functions on $\Omega$ which are $r$-times differentiable with derivatives of order $r$ being $\alpha$-Hölder continuous functions. To avoid boundary problems, we take $C^{r, \alpha}(\Omega)$ to contain only functions $f$ whose derivatives of order $r$ are locally Hölder continuous, i.e., for each point $\mathbf{z} \in \Omega$, there is an open ball $B$ contained in $\Omega$, with center $\mathbf{z}$, such that the Hölder seminorm

$$
\sup _{\mathbf{x}, \mathbf{y} \in B, \mathbf{x} \neq \mathbf{y}} \frac{\left|\partial^{\gamma} f(\mathbf{x})-\partial^{\gamma} f(\mathbf{y})\right|}{|\mathbf{x}-\mathbf{y}|^{\alpha}}<\infty,
$$

for all multi-indices $\boldsymbol{\gamma}$ with $|\boldsymbol{\gamma}|=r$. For $r \in \mathbf{N}_{0}$ and $\alpha \in\langle 0,1\rangle$, we denote by $c^{r, \alpha}(\Omega)$ the vector subspace of $C^{r, \alpha}(\Omega)$ containing functions $f$ which have the stronger property that there exists a family of balls (which may depend on $f$ ) covering $\Omega$ such that for each ball $B$ in this family

$$
\lim _{h \rightarrow 0} \sup _{\substack{|\mathbf{x}-\mathbf{y}|<h, \mathbf{x}, \mathbf{y} \in B, \mathbf{x} \neq \mathbf{y}}} \frac{\left|\partial^{\gamma} f(\mathbf{x})-\partial^{\gamma} f(\mathbf{y})\right|}{|\mathbf{x}-\mathbf{y}|^{\alpha}}=0,
$$

for all multi-indices $\boldsymbol{\gamma}$ with $|\boldsymbol{\gamma}|=r$. We also denote by $C^{r}(\Omega)=c^{r, 0}(\Omega)$ the vector space of $r$-times continuously differentiable functions. All of the above introduced spaces shall be considered without a topology.

For $r \in \mathbf{N}_{0}, \alpha \in\langle 0,1]$, and a compact set $K \subset \Omega$ denote by $C_{K}^{r, \alpha}(\Omega)$ the set of all $C^{r, \alpha}(\Omega)$ functions whose support is contained in $K$. Since $K$ is compact, $C_{K}^{r, \alpha}(\Omega)$ is a Banach space with norm given by

$$
\|f\|_{C_{K}^{r, \alpha}(\Omega)}:=\sum_{|\gamma| \leq r}\left\|\partial^{\gamma} f\right\|_{L^{\infty}(\Omega)}+\sum_{|\gamma|=r}[f]_{C^{\gamma, \alpha}(\Omega)}
$$

where

$$
[f]_{C^{\gamma, \alpha}(\Omega)}:=\sup _{\mathbf{x}, \mathbf{y} \in \Omega, \mathbf{x} \neq \mathbf{y}} \frac{\left|\partial^{\gamma} f(\mathbf{x})-\partial^{\gamma} f(\mathbf{y})\right|}{|\mathbf{x}-\mathbf{y}|^{\alpha}}<\infty,
$$

and thus, it is a locally convex space. We can take the same norms for the spaces $c^{r, \alpha}(\Omega)$ for $r \in \mathbf{N}_{0}$ and $\alpha \in[0,1\rangle$, i.e., we define $\|f\|_{C_{K}^{r, \alpha}(\Omega)}:=\|f\|_{C_{K}^{r, \alpha}(\Omega)}$ and $[f]_{c^{\gamma, \alpha}(\Omega)}:=[f]_{C^{\gamma, \alpha}(\Omega)}$. Given two compact sets $K$ and $L$ such that Int $K \subset L$, it holds $C_{K}^{0, \alpha+\varepsilon}(\Omega) \subset C_{L}^{0, \alpha}(\Omega)$ for all $\varepsilon$ sufficiently small, the embedding being continuous and compact. The same is true for the embedding $c_{K}^{0, \alpha+\varepsilon}(\Omega) \subset c_{L}^{0, \alpha}(\Omega)$.

If $\varepsilon$ is sufficiently small, one can approximate each function $f \in C_{K}^{r, \alpha+\varepsilon}(\Omega)$ by a $C_{L}^{\infty}(\Omega)$ function (Int $K \subset L$ ) by using a mollifier, but in the norm of $C_{L}^{r, \alpha}(\Omega)$. On the other hand, as is also well known, one cannot approximate $C_{K}^{r, \alpha}(\Omega)$ functions by compactly supported smooth functions in the norm of $C_{L}^{r, \alpha}(\Omega)$. The obstruction which occurs is that if one takes a slightly more smooth function $g$ than the functions in $C_{K}^{r, \alpha}(\Omega)$, one has (if $\alpha<1$ and $|\gamma|=r)$

$$
\lim _{|\mathbf{x}-\mathbf{y}| \rightarrow 0, \mathbf{x} \neq \mathbf{y}} \frac{\left|\partial^{\gamma} g(\mathbf{x})-\partial^{\gamma} g(\mathbf{y})\right|}{|\mathbf{x}-\mathbf{y}|^{\alpha}}=0,
$$

and hence, any limit of smooth functions in the norm of $C_{K}^{r, \alpha}(\Omega)$ has this property too. This is just the fact that $c_{K}^{r, \alpha}(\Omega)$ is a strict Banach subspace of $C_{K}^{r, \alpha}(\Omega)$. In fact, we can approximate 
$c_{K}^{r, \alpha}(\Omega)$ functions with $C_{L}^{\infty}(\Omega)$ functions in the norm of $c_{L}^{r, \alpha}(\Omega)$. For more on Hölder spaces, we refer the reader to $[1,15]$.

We shall introduce three families of Hölder test function spaces. We start with the first two.

Definition 1 Let $\left(K_{n}\right)$ be an increasing sequence of compact subsets of $\Omega$ which exhaust it, i.e., $\Omega=\cup_{n \in \mathbf{N}} K_{n}$ and $K_{n} \subset$ Int $K_{n+1}$.

We define the space $C_{c}^{r, \alpha}(\Omega), r \in \mathbf{N}_{0}, 0<\alpha \leq 1$, as the space of all $C^{r, \alpha}(\Omega)$ functions with compact support, and equip it with the strict inductive limit topology generated by the natural inclusions $C_{K_{n}}^{r, \alpha}(\Omega) \rightarrow C_{c}^{r, \alpha}(\Omega)$.

We define second space $c_{c}^{r, \alpha}(\Omega), r \in \mathbf{N}_{0}, 0 \leq \alpha<1$, as the space of all $c^{r, \alpha}(\Omega)$ functions with compact support, and also equip it with the strict inductive limit topology generated by the natural inclusions $c_{K_{n}}^{r, \alpha}(\Omega) \rightarrow c_{c}^{r, \alpha}(\Omega)$. In the case $\alpha=0$, we also denote this space with $C_{c}^{r}(\Omega)$

Before introducing the third family of spaces, we elaborate on some properties of the spaces $C_{c}^{r, \alpha}(\Omega)$ and $c_{c}^{r, \alpha}(\Omega)$. Recall that $C_{c}^{r, \alpha}(\Omega)$ (resp. $\left.c_{K_{n}}^{r, \alpha}(\Omega)\right)$ is a Hausdorff, complete space as a strict inductive limit of such spaces. The space $C_{c}^{\infty}(\Omega)$ embeds continuously into $C_{c}^{r, \alpha}(\Omega)$, though not densely. On the other hand, $c_{c}^{r, \alpha}(\Omega)$ has $C_{c}^{\infty}(\Omega)$ as a dense subspace, and so it is separable. It is the case that a subset of $C_{c}^{r, \alpha}(\Omega)\left(\operatorname{resp} . c_{c}^{r, \alpha}(\Omega)\right)$ is bounded if and only if it is contained and bounded in some $C_{K_{n}}^{r, \alpha}(\Omega)\left(\right.$ resp. $\left.c_{K_{n}}^{r, \alpha}(\Omega)\right)$. This is called the DieudonnéSchwartz theorem. See, e.g., [22] or [25]. From this, it follows that $C_{c}^{r, \alpha}(\Omega)\left(\operatorname{resp} . c_{c}^{r, \alpha}(\Omega)\right)$ is not a Montel space since closed and bounded sets are not compact in the Banach space $C_{K_{n}}^{r, \alpha}(\Omega)\left(\right.$ resp. $c_{K_{n}}^{r, \alpha}(\Omega)$ ). In fact, $C_{c}^{r, \alpha}(\Omega)$ and $c_{c}^{r, \alpha}(\Omega)$ are not even semireflexive. Namely, if they were semireflexive, then also their respective closed subspaces $C_{K_{n}}^{r, \alpha}(\Omega)$ and $c_{K_{n}}^{r, \alpha}(\Omega)$ would be semireflexive (this follows from [22, page 144, 5.5, d)]), and hence also reflexive since Banach spaces are barrelled. But since Hölder spaces are not reflexive, this gives a contradiction.

Now, since $C_{K_{n}}^{r, \alpha}(\Omega)$ are not separable, one may ask whether $C_{c}^{r, \alpha}(\Omega)$ is separable. The fact that an inductive limit of countably many separable spaces is again separable is well known and easily proven. We are interested in a converse of this statement. Notably, separability is not well behaved for general topological spaces, e.g., non-open subspaces of separable topological spaces (which are not metrizable, but otherwise they can be well behaved) are not generally separable. This also extends to topological vector spaces: There exist separable topological vector spaces with dense or closed (even complete) non-separable subspaces (see $[10,13,18]$ ). Despite this, we also have (see [18]) that metrizable subspaces of separable spaces are again separable. Therefore, $C_{c}^{r, \alpha}(\Omega)$ is not separable. We collect the above (essentially known) observations in a proposition.

Proposition 2 The spaces $c_{c}^{r, \alpha}(\Omega)$ and $C_{c}^{r, \alpha}(\Omega)$ are neither Montel nor semireflexive. The spaces $c_{c}^{r, \alpha}(\Omega)$ are separable, and the spaces $C_{c}^{r, \alpha}(\Omega)$ are not. $c_{c}^{r, \alpha}(\Omega)$ have $C_{c}^{\infty}(\Omega)$ as a dense subspace.

The most interesting family of spaces is the following one.

Definition 3 Let $\left(K_{n}\right)$ be an increasing sequence of compact subsets such that $\Omega=\cup_{n \in \mathbf{N}} K_{n}$ and $K_{n} \subset$ Int $K_{n+1}$. We define $C_{c}^{r, \alpha+}(\Omega), r \in \mathbf{N}_{0}, 0 \leq \alpha<1$ as the space of all $c^{r, \alpha+\varepsilon}(\Omega)$, $0<\varepsilon<1-\alpha$, functions with compact support, and equip it with the inductive limit topology (note this inductive limit is not strict) generated by the natural inclusions $c_{K_{n}}^{r, \alpha+1 / n}(\Omega) \rightarrow$ $\mathrm{C}_{c}^{r, \alpha+}(\Omega)$, i.e., the finest locally convex topology such that all the inclusions are continuous. One can also define this space using the spaces $C_{K_{n}}^{r, \alpha+1 / n}(\Omega)$. 
One should notice that the space $C_{c}^{\infty}(\Omega)$ embeds continuously and densely into $C_{c}^{r, \alpha+}(\Omega)$, which can be seen using say a mollifier. In fact, the spaces $C_{c}^{r, \alpha+}(\Omega), r \in \mathbf{N}_{0}, 0 \leq \alpha<1$, have much better properties than either of the spaces $C_{c}^{r, \alpha}(\Omega), r \in \mathbf{N}_{0}, 0<\alpha \leq 1$, or $c_{c}^{r, \alpha}(\Omega), r \in \mathbf{N}_{0}, 0 \leq \alpha<1$, the reason being that the embeddings

$$
\iota_{n, n+1}: C_{K_{n}}^{r, \alpha+1 / n}(\Omega) \rightarrow \mathrm{C}_{K_{n+1}}^{r, \alpha+1 /(n+1)}(\Omega)
$$

are compact. We use the general theorems from the article [17, Theorem 6', Theorem 7'] which we adopt to our setting.

Theorem 4 For the space $C_{c}^{r, \alpha+}(\Omega), 0 \leq \alpha<1$, the following is valid.

(a) It is a separable Hausdorff complete bornological (DF) Montel space. In particular, it is reflexive, barrelled, and webbed.

(b) If $B$ is a bounded subset of $\mathrm{C}_{c}^{r, \alpha+}(\Omega)$, then it is a subset of $\mathrm{C}_{K_{n}}^{r, \alpha+1 / n}(\Omega)$ for some $n \in N$ and it is bounded in its Banach space topology. Also, on $B$ the inductive topology and the weak topology coincide.

(c) A sequence $\left(f_{n}\right)$ converges in $C_{c}^{r, \alpha+}(\Omega)$ if and only if it is contained in $C_{K_{n}}^{r, \alpha+1 / n}(\Omega)$ for some $n \in N$ and it converges in its Banach space topology.

(d) The inductive limit topology coincides with the general (non-locally convex) inductive limit topology.

(e) If $Z$ is a closed subspace of $\mathrm{C}_{c}^{r, \alpha+}(\Omega)$, then its subspace topology coincides with the topology of the inductive limit of spaces $Z \cap \mathrm{C}_{K_{n}}^{r, \alpha+1 / n}(\Omega), n \in \mathbf{N}$.

Proof Hausdorffness follows from [17, Lemma 3]. The rest of the first statement in (a) is precisely the first statement in [17, Theorem 6']. Recall that all Montel spaces are reflexive and barrelled. The fact that it is webbed follows from the fact that it is reflexive and its strong dual is a Fréchet space (this we shall see explicitly in Theorem 13), and so we can apply [4, Theorem 14.6.4].

The statements in (b), (c), and (d) are all contained in [17, Theorem 6']. The part (e) is also just an application of [17, Theorem 7'] to our case.

Remark 5 The fact that $C_{c}^{r, \alpha+}(\Omega)$ is Hausdorff, separable, complete, barrelled, and bornological can alternatively be seen by using more elementary properties of (strict) inductive limits. Separability follows by using either the density of compactly supported smooth functions, or the fact that $\mathrm{C}_{c}^{r, \alpha+}(\Omega)$ is a countable union of separable compact sets. The fact that it is barrelled and bornological follows easily from the fact that each $C_{K_{n}}^{r, \alpha+1 / n}(\Omega)$ is such as a Banach space, and inductive limits inherit these properties. We give sketches of alternative proofs of Hausdorffness and completeness which are in a certain sense extrinsic. Hausdorffness follows from the fact that the embedding of $\mathrm{C}_{c}^{r, \alpha+}(\Omega)$ into $c_{c}^{r, \alpha}(\Omega)$ is continuous, and using the fact that $c_{c}^{r, \alpha}(\Omega)$ is Hausdorff as a strict inductive limit.

The alternative argument for completeness is slightly more involved. Assume there exists a Cauchy net which does not converge in $\mathrm{C}_{c}^{r, \alpha+}(\Omega)$. As the embedding of $\mathrm{C}_{c}^{r, \alpha+}(\Omega)$ into $c_{c}^{r, \alpha}(\Omega)$ is continuous, then the same net converges in $\mathrm{c}_{c}^{r, \alpha}(\Omega)$ in its topology (since this is a strict inductive limit topology) to an element $\varphi \notin \mathrm{C}_{c}^{r, \alpha+}(\Omega)$. Therefore, if we now denote

$$
\omega_{\varphi}(h):=\sup _{|\boldsymbol{\gamma}|=r,|\mathbf{x}-\mathbf{y}| \leq h}\left|\partial^{\gamma} \varphi(\mathbf{x})-\partial^{\gamma} \varphi(\mathbf{y})\right|,
$$

then for each $n$ there exists a sequence $\left(h_{k}^{n}\right)$ strictly decreasing to 0 such that

$$
\omega_{\varphi}\left(h_{k}^{n}\right) /\left(h_{k}^{n}\right)^{\alpha+1 / n} \rightarrow+\infty .
$$


Using this, one can easily obtain a sequence $\left(h_{k}\right)$ strictly decreasing to 0 such that $\sup _{k} h_{k}<1$ and

$$
\omega_{\varphi}\left(h_{k}\right) \geq\left(h_{k}\right)^{\alpha+1 / k}
$$

Now we construct an intermediary space $\mathrm{C}_{c}^{r, \omega}(\Omega)$ of compactly supported functions whose $r$ th derivatives have modulus of continuity slightly stronger by a logarithmic factor than the modulus of the $r$ th derivatives of the function $\varphi$, i.e.,

$$
\omega(h):=\frac{-1}{\ln (\min \{h, 1 / 2\})} \sup _{\left\{k: h_{k+1}<h\right\}} \omega_{\varphi}\left(h_{k}\right) .
$$

We endow $C_{c}^{r, \omega}(\Omega)$ with a strict inductive topology as usual, and so it is complete. Now note that for $h \in\left\langle h_{k+1}, h_{k}\right]$ we have $\omega(h) \gtrsim \varepsilon h^{\varepsilon} h^{\alpha+1 / k}$ for $\varepsilon$ small. In particular if $h \leq h_{k}$ and $\varepsilon+1 / k \leq 1 / n$, then $\omega(h) \gtrsim_{n} h^{\alpha+1 / n}$. Therefore, for each $n$ the space the space $C_{K_{n}}^{r, \alpha+1 / n}(\Omega)$ embeds into $C_{c}^{r, \omega}(\Omega)$ continuously, and hence, $\mathrm{C}_{c}^{r, \alpha+}(\Omega)$ embeds continuously into $\mathrm{C}_{c}^{r, \omega}(\Omega)$ too. But now on one hand $\varphi$ is not an element of this space by construction, and on the other, it has to be since the space $C_{c}^{r, \alpha+}(\Omega)$ embeds continuously into it and hence the given net must converge in it, a contradiction.

We know that the space $C_{c}^{r, \alpha+}(\Omega)$ is Montel, so the next natural question to ask is whether this space has the stronger property of being nuclear. The answer is no; this will be proved in Sect. 4.

\section{Distributions of positive real order}

Definition 6 A Hölder distribution of order smaller or equal to $r+\alpha, r \in \mathbf{N}_{0}, 0 \leq \alpha<1$, is any continuous linear functional on $c_{c}^{r, \alpha}(\Omega)$. We denote the space of all such functionals by $\mathcal{D}_{r+\alpha}^{\prime}(\Omega)$.

Remark 7 Note that for a distribution $T$, satisfying the bound

$$
|\langle T, \varphi\rangle| \leq C_{K}\|\varphi\|_{C_{K}^{r, 1}(\Omega)},
$$

for all $\varphi \in C_{K}^{\infty}(\Omega)$ and compact $K$ contained in $\Omega$ is equivalent to $T$ being a distribution of classical order at most $r+1$ since the Lipschitz norm is equivalent to the $C^{1}$ norm.

As the space $C_{c}^{\infty}(\Omega)$ is not dense in $\mathrm{C}_{c}^{r, \alpha}(\Omega), 0<\alpha \leq 1$, we cannot naturally extend the just defined distributions to functionals on $\mathrm{C}_{c}^{r, \alpha}(\Omega)$. Instead, for $0<\alpha<1$, we view them as functionals on the closure of $C_{c}^{\infty}(\Omega)$ in the topology of $C_{c}^{r, \alpha}(\Omega)$, which is precisely the space $c_{c}^{r, \alpha}(\Omega)$. Note that $\mathcal{D}_{r+\alpha}^{\prime}(\Omega)$ is a Fréchet space with the strong topology as a dual of a strict inductive limit of Banach spaces. This is obvious from the fact that the Dieudonné-Schwartz theorem is valid for $\mathrm{c}_{c}^{r, \alpha}(\Omega)$.

The following spaces are much more suitable from the viewpoint of functional analysis.

Definition 8 A Hölder distribution of order smaller or equal to $(r+\alpha)+, r \in \mathbf{N}_{0}, 0 \leq \alpha<1$, is any continuous linear functional on $C_{c}^{r, \alpha+}(\Omega)$. We denote the space of all such functionals by $\mathcal{D}_{(r+\alpha)+}^{\prime}(\Omega)$.

Indeed, each Hölder distribution from $\mathcal{D}_{(r+\alpha)+}^{\prime}(\Omega)$ is a distribution in the classical sense since $C_{c}^{\infty}(\Omega)$ is dense in the space $C_{c}^{r, \alpha+}(\Omega)$ for each $0 \leq \alpha<1$. 
For $0 \leq \beta<\alpha$, we have that every Hölder distribution of order at most $\beta$ is a Hölder distribution of order at most $\beta+$, and every Hölder distribution of order at most $\beta+$ is a Hölder distribution of order at most $\alpha$. Furthermore, it is not true that a Hölder distribution of order at most $0+$ is a distribution of classical order 0 ; we shall give a simple example later. The same can be shown for $\alpha+$ and $\alpha$ when $\alpha \in \mathbf{R}_{>0}$.

Definition 9 The order of a Hölder distribution is the smallest order for which we have continuity in the corresponding topologies.

Remark 10 This is well defined since it follows from the property of inductive limits that a distribution $T$ is continuous on $\mathrm{C}_{c}^{r, \alpha+}(\Omega)$ if and only if it is continuous with respect to the topology of $\mathrm{c}_{c}^{0, \alpha+\varepsilon}(\Omega)$ for all $\varepsilon>0$ sufficiently small. Therefore, the spaces $\mathrm{C}_{c}^{r, \alpha+}(\Omega)$ are also quite natural to consider when defining the continuous order of a distribution.

The following criteria hold, similarly to classical distributions.

Proposition 11 For a linear functional $u$ on $c_{c}^{r, \alpha}(\Omega)$, the following are equivalent

(a) $u \in \mathcal{D}_{r+\alpha}^{\prime}(\Omega)$,

(b) $(\forall K \subset \Omega$ compact $)\left(\exists C_{K}>0\right)\left(\forall \varphi \in c_{K}^{r, \alpha}(\Omega)\right)$

$$
|\langle u, \varphi\rangle| \leq C_{K}\|\varphi\|_{\mathrm{c}_{K}^{r, \alpha}},
$$

(c) $(\forall K \subset \Omega$ compact $)\left(\exists C_{K}>0\right)\left(\forall \varphi \in C_{K}^{\infty}(\Omega)\right)$

$$
|\langle u, \varphi\rangle| \leq C_{K}\|\varphi\|_{\mathrm{c}_{K}^{r, \alpha}},
$$

(d) for every sequence $\left(\varphi_{k}\right)$ converging to zero in $c_{c}^{r, \alpha}(\Omega)$, the scalar sequence $\left(\left\langle u, \varphi_{k}\right\rangle\right)$ converges to zero.

(e) for each $n \in \mathbf{N}$ and for every sequence $\left(\varphi_{k}\right)$ converging to zero in $c_{K_{n}}^{r, \alpha}(\Omega)$, the scalar sequence $\left(\left\langle u, \varphi_{k}\right\rangle\right)$ converges to zero.

We omit the proof completely since it is the same as the proof for classical distributions of finite order as the spaces $\mathrm{c}_{c}^{r, \alpha}(\Omega)$ have the same properties as the spaces $C_{c}^{r}(\Omega)$. In fact, the proof is almost the same as the proof of the following analogous proposition for $\mathcal{D}_{(r+\alpha)+}^{\prime}(\Omega)$.

Proposition 12 For a linear functional u on $C_{c}^{r, \alpha+}(\Omega)$, the following are equivalent

(a) $u \in \mathcal{D}_{(r+\alpha)+}^{\prime}(\Omega)$,

(b) $(\forall K \subset \Omega$ compact $)(\forall \varepsilon>0$ small $)\left(\exists C_{K, \varepsilon}>0\right)\left(\forall \varphi \in \mathrm{c}_{K}^{r, \alpha+\varepsilon}(\Omega)\right)$

$$
|\langle u, \varphi\rangle| \leq C_{K, \varepsilon}\|\varphi\|_{c_{K}^{r, \alpha+\varepsilon}}
$$

(c) $(\forall K \subset \Omega$ compact $)(\forall \varepsilon>0$ small $)\left(\exists C_{K, \varepsilon}>0\right)\left(\forall \varphi \in C_{K}^{\infty}(\Omega)\right)$

$$
|\langle u, \varphi\rangle| \leq C_{K, \varepsilon}\|\varphi\|_{c_{K}^{r, \alpha+\varepsilon}}
$$

(d) for every sequence $\left(\varphi_{k}\right)$ converging to zero in $c_{c}^{r, \alpha+}(\Omega)$ the scalar sequence $\left(\left\langle u, \varphi_{k}\right\rangle\right)$ converges to zero.

(e) for each $n \in \mathbf{N}$ and for every sequence $\left(\varphi_{k}\right)$ converging to zero in $c_{K_{n}}^{r, \alpha+1 / n}(\Omega)$ the scalar sequence $\left(\left\langle u, \varphi_{k}\right\rangle\right)$ converges to zero. 
Proof The equivalence (a) $\Longleftrightarrow$ (b) follows from the fact that a functional $u$ is continuous on $\mathrm{c}_{c}^{r, \alpha+}(\Omega)$ if and only if it is continuous for each $n$ on $c_{K_{n}}^{r, \alpha+1 / n}(\Omega)$. The equivalence (b) $\Longleftrightarrow$ (c) is just the density of $\mathrm{C}^{\infty}(\Omega)$ in $c^{r, \alpha+\varepsilon}(\Omega)$. The implication (a) $\Longrightarrow$ (d) is trivial. The implication (d) $\Longrightarrow$ (e) follows from the fact that $\mathrm{c}_{K_{n}}^{r, \alpha+1 / n}(\Omega)$ embeds continuously into $\mathrm{c}_{c}^{r, \alpha+}(\Omega)$. Finally, the implication e) $\Longrightarrow$ (b) follows from the fact $\mathrm{c}_{K}^{r, \alpha+\varepsilon}(\Omega)$ ) embeds continuously into $\mathrm{c}_{K_{n}}^{r, \alpha+1 / n}(\Omega)$ for $n$ sufficiently large, and the fact that $c_{K}^{r, \alpha+\varepsilon}(\Omega)$ ) is a Banach space where sequential continuity implies continuity.

Next we give some properties of the space $u \in \mathcal{D}_{(r+\alpha)+}^{\prime}(\Omega)$.

Theorem 13 The space $\mathcal{D}_{(r+\alpha)+}^{\prime}(\Omega)$ with the strong topology is reflexive and a FréchetSchwartz space, it is a projective limit of spaces $\left(\mathrm{c}_{K_{n}}^{r, \alpha+1 / n}(\Omega)\right)^{\prime}$, and its topology is generated by the increasing sequence of seminorms

$$
\begin{aligned}
&\|T\|_{n}:= \sup _{\substack{\varphi \in c_{K_{n}}^{r, \alpha+1 / n}(\Omega) \\
\|\varphi\|_{c_{K_{n}}^{r, \alpha+1 / n}(\Omega)}}}|T(\varphi)| \\
&=\|T\|_{\left(c_{K_{n}}^{r, \alpha+1 / n}(\Omega)\right)^{\prime}}
\end{aligned}
$$

Proof First note that because of Theorem 4 it follows that $\mathcal{D}_{(r+\alpha)+}^{\prime}(\Omega)$ is reflexive, and we know that $c_{c}^{r, \alpha+}(\Omega)$ is a complete (DF) space. Therefore, we can apply [17, Theorem 18, c)] to obtain that $\mathcal{D}_{(r+\alpha)+}^{\prime}(\Omega)$ with the strong topology is a compact projective limit of a sequence, which, as mentioned in [17, Remark 6], means it is a Fréchet-Schwartz space. [17, Theorem 12] identifies $\left(c_{K_{n}}^{r, \alpha+1 / n}(\Omega)\right)^{\prime}$ as the sequence of the projective limit. For the last claim, one just needs to recall that the strong topology is the topology of uniform convergence on bounded sets. Namely, by the Dieudonné-Schwartz theorem (which is valid for $c_{c}^{r, \alpha+}(\Omega)$ by Theorem 4), each bounded set can be absorbed by a set of the form $\left\{\varphi \in c_{K_{n}}^{r, \alpha+1 / n}(\Omega):\|\varphi\|_{c_{K_{n}(\Omega)}^{r, \alpha+1 / n}} \leq 1\right\}$, and so in particular, the norms $\|\cdot\|_{n}$ completely determine the strong topology.

Remark 14 Let us mention three properties of Hölder distributions of order $0+$ that classical distributions do not generally possess. First, one can define Hölder distributions of order at most $0+$ (and even less than 1) on general metric spaces, as there Hölder functions are well defined. The second property is that Hölder distributions of order $0+$ are completely determined by restriction to their support (see the proof of [5, Lemma 1]). This is a property that Radon measures have, but some other distributions as $\delta^{\prime}$ do not. It proved to be crucial in Bonahon's work. And third, if $Y$ is a closed subset of $X$, then every $0+$ Hölder distribution $T$ on $Y$ (in other words, $T$ has support in $Y$ in accordance with the restriction property we just mentioned) can be easily extended to a $0+$ Hölder distribution $\bar{T}$ on the whole $X$ by simply setting $\langle\bar{T}, \varphi\rangle=\left\langle T, \varphi_{\left.\right|_{Y}}\right\rangle$. This correspondence between $0+$ distributions on $Y$ and $0+$ distributions on $X$ with support in $Y$ is actually one to one (see the proof of [5, Lemma 2]).

\section{Proof of non-nuclearity of $C_{c}^{r, \alpha+}(\Omega)$ and $\mathcal{D}_{(r+\alpha)+}^{\prime}(\Omega)$}

In this section, we prove the following theorem.

Theorem 15 The spaces $C_{c}^{r, \alpha+}(\Omega)$ and $\mathcal{D}_{(r+\alpha)+}^{\prime}(\Omega), r \in \mathbf{N}_{0}, 0 \leq \alpha<1$, are not nuclear. 
Since $C_{c}^{r, \alpha+}(\Omega)$ is nuclear if and only if $\mathcal{D}_{(r+\alpha)+}^{\prime}(\Omega)$ is nuclear (see [25, page 523, Proposition 50.6.]), it is sufficient to prove that the Fréchet space $\mathcal{D}_{(r+\alpha)+}^{\prime}(\Omega)$ is not nuclear. First we shall prove this for the space $\mathcal{D}_{(r+\alpha)+}^{\prime}(\mathbb{T})$, where $\mathbb{T}$ is the one-dimensional torus (i.e., the circle), and later we shall see how the general case easily follows from this one. We start with an auxiliary result from classical Fourier analysis.

Lemma 16 Consider a function $f \in c^{0, \varepsilon}(\mathbb{T}), 0<\varepsilon<1$ and denote by $S_{N}$ the Nth Fourier partial sum operator. Then,

$$
\left\|S_{N} f-f\right\|_{L^{\infty}(\mathbb{T})} \lesssim\|f\|_{\mathrm{c}^{0, \varepsilon}(\mathbb{T})} \frac{\ln N}{N^{\varepsilon}} .
$$

For a proof, see [3, Chapter IV, Section 4] and references therein.

The following lemma gives us a result interesting in itself. The same result does not hold for classical Hölder spaces.

Lemma 17 The sequence $\left(x \mapsto e^{2 \pi i k x}\right)_{k \in \mathbf{Z}}$ is a Schauder basis for $C^{r, \alpha+}(\mathbb{T}), r \in \mathbf{N}_{0}$, $0 \leq \alpha<1$. In fact,

$$
\lim _{N \rightarrow \infty} \sup _{\|f\|_{c^{r, \alpha+1 / n}(\mathbb{T})} \leq 1}\left\|S_{N} f-f\right\|_{c^{r, \alpha+1 / m}(\mathbb{T})}=0
$$

for all $m>n>1 /(1-\alpha)$. Here $S_{N}$ denotes the partial sum operator, i.e., $S_{N} f=D_{N} * f$, where $D_{N}$ is the Dirichlet kernel.

Proof Since the differential operator $\partial^{r}$ commutes with $S_{N}$, it is sufficient to prove the theorem for $r=0$. The convergence of the Hölder seminorm follows from the bound

$$
\begin{aligned}
|f(x)-f(y)-f(x-t)+f(y-t)| & \leq 2\|f\|_{c^{0, \alpha+1 / n}(\mathbb{T})} \min \left\{|x-y|^{\alpha+1 / n},|t|^{\alpha+1 / n}\right\} \\
& \leq 2\|f\|_{c^{0, \alpha+1 / n}(\mathbb{T})}|x-y|^{\alpha+1 / m}|t|^{1 / n-1 / m},
\end{aligned}
$$

valid for $f \in c^{0, \alpha+1 / n}(\mathbb{T})$. One just needs to apply Lemma 16 to $t \mapsto f(t)$ with $\varepsilon<1 / n$ in order to obtain $L^{\infty}$ convergence, and to $t \mapsto f(x)-f(y)-f(x-t)+f(y-t)$ with $\varepsilon=1 / n-1 / m$ in order to obtain convergence of the $\mathrm{c}^{0, \alpha+1 / m}(\mathbb{T})$ seminorm.

Corollary 18 The sequence $\left(x \mapsto e^{2 \pi i n x}\right)_{n \in \mathbf{Z}}$ is a Schauder basis for $\mathcal{D}_{(r+\alpha)+}^{\prime}(\mathbb{T})$, in the strong topology.

Proof Since $S_{N}$ is self-adjoint, one has

$$
\left\langle S_{N} T-T, f\right\rangle=\left\langle T, S_{N} f-f\right\rangle
$$

and so the claim follows by Lemma 17.

We begin with the proof of Theorem 15.

Step 1 Proof that $\mathcal{D}_{(r+\alpha)+}^{\prime}(\mathbb{T})$ is not nuclear.

If $\mathcal{D}_{(r+\alpha)+}^{\prime}(\mathbb{T})$ were a nuclear space, then also its associated Köthe space would be nuclear (see, e.g., [11]). We shall prove that this is not the case. We denote by $e_{n}(x)=e^{2 \pi i n x}$ the elements of the Schauder basis of $\mathcal{D}_{(r+\alpha)+}^{\prime}(\mathbb{T})$, and by $\|\cdot\|_{k}$ the seminorm $\|\cdot\|_{\left(\mathrm{c}_{K_{n}}^{r, \alpha+1 / k}(\mathbb{T})\right)^{\prime}}$ of $\mathcal{D}_{(r+\alpha)+}^{\prime}(\mathbb{T})$. Define $a_{n}^{k}=\left\|e_{n}\right\|_{k}$. Then, the associated Köthe space is defined as

$$
K(a)=\left\{\xi=(\xi)_{n \in \mathbf{Z}}:|\xi|_{k}=\sum_{n \in \mathbf{Z}}\left|\xi_{n}\right| a_{n}^{k}<\infty\right\},
$$


with seminorms $|\cdot|_{k}$ which make it into a Fréchet space. If $\mathcal{D}_{(r+\alpha)+}^{\prime}(\mathbb{T})$ were nuclear, then the Köthe space would also be nuclear and it would satisfy the condition

$$
(\forall k \in \mathbf{N})(\exists j \in \mathbf{N}) \sum_{n \in \mathbf{Z}} \frac{a_{n}^{k}}{a_{n}^{j}}<\infty .
$$

Let us calculate

$$
\begin{aligned}
a_{n}^{k}=\left\|e_{n}\right\|_{k} & =\sup _{\substack{\varphi \in c^{r, \alpha+1 / k}(\mathbb{T}) \\
\|\varphi\|_{c^{r, \alpha+1 / k}(\mathbb{T})} \leq 1}}\left|e_{n}(\varphi)\right| \\
& =\sup _{\substack{\varphi \in c^{r, \alpha+1 / k}(\mathbb{T}) \\
\|\varphi\|_{c^{r, \alpha+1 / k}(\mathbb{T})} \leq 1}}|\hat{\varphi}(n)|,
\end{aligned}
$$

and therefore $\left|a_{n}^{k}\right| \lesssim|n|^{-(r+\alpha+1 / k)}$ for some $C>0$ since this is a well-known decay property of the Fourier coefficients of Hölder continuous functions (see, e.g., [14] or [27]). For an estimate below of $\left|a_{n}^{k}\right|$, we just need a rough one (actually the upper bound is also stronger than needed), obtained by considering $\varphi=e_{n}$. Therefore, we have

$$
\left|a_{n}^{k}\right| \geq \frac{1}{\left\|e_{n}\right\|_{c^{r, \alpha+1 / k}(\mathbb{T})}} \gtrsim \frac{1}{\left\|e_{n}\right\|_{C^{r+1}(\mathbb{T})}} \gtrsim \frac{1}{|n|^{r+1}} .
$$

for $n \neq 0$ and some $C>0$. Finally, we see that for all $k, j \in \mathbf{N}$

$$
\sum_{n \in \mathbf{Z}} \frac{a_{n}^{k}}{a_{n}^{j}} \gtrsim \sum_{n \in \mathbf{Z} \backslash\{0\}} \frac{|n|^{r+\alpha+1 / j}}{|n|^{r+1}}=\infty
$$

and so the condition (1) is not satisfied. Therefore, $\mathcal{D}_{(r+\alpha)+}^{\prime}(\mathbb{T})$ cannot be nuclear, and so the same is true for $\mathrm{C}^{r, \alpha+}(\mathbb{T})$.

Step 2 Proof that $C_{c}^{r, \alpha+}(\Omega)$ is not nuclear for open sets $\Omega \subseteq \mathbf{R}^{d}$ when $d>1$.

Let $\Omega \subseteq \mathbf{R}^{d}$ be an open set with $d>1$, and consider $\mathbb{T}$ as a (compact) submanifold of $\Omega$ (this obviously cannot be done for $d=1$ ). Note that the restriction map $\pi: C_{c}^{r, \alpha+}(\Omega) \rightarrow$ $\mathrm{C}^{r, \alpha+}(\mathbb{T})$ is continuous and surjective since it is continuous and surjective as a map $\pi$ : $\mathrm{c}_{K_{k}}^{r, \alpha+1 / k}(\Omega) \rightarrow \mathrm{c}^{\alpha+1 / k}(\mathbb{T})$ for all $k \in \mathbf{N}$ large enough. By an open mapping theorem (see [12, page 450, Theorem 6.7.2]), $\pi: C_{c}^{r, \alpha+}(\Omega) \rightarrow C^{r, \alpha+}(\mathbb{T})$ is an open mapping. We conclude that $C^{r, \alpha+}(\mathbb{T})$ is isomorphic as a topological vector space to a quotient space of $C_{c}^{r, \alpha+}(\Omega)$. Hence, $C_{c}^{r, \alpha+}(\Omega)$ is not nuclear since quotients (by closed subspaces) of nuclear spaces are nuclear.

Step 3 Proof that $C_{c}^{r, \alpha+}(\Omega)$ is not nuclear for open sets $\Omega \subseteq \mathbf{R}$.

In the one-dimensional case, we have exactly the same proof as in the higher dimensions, except we use a different quotient map. Note that it is sufficient to consider only $\Omega=\mathbf{R}$ since open intervals are diffeomorphic to $\mathbf{R}$. For general open sets, non-nuclearity follows from the interval case since open sets of $\mathbf{R}$ are disjoint unions of open intervals, and restriction to one such interval gives a quotient map.

The quotient map we use in the case $\Omega=\mathbf{R}$ is the periodization map defined on the space $C_{c}^{r, \alpha+}(\mathbf{R})$ and given by

$$
P \varphi(x)=\sum_{l \in \mathbf{Z}} \varphi(x+l) .
$$

Since the function $\varphi$ is compactly supported, the above sum is finite for each $x \in \mathbf{R}$ In fact, the above sum is finite for each bounded subset of $\mathbf{R}$, and from this, one easily sees 
that $\mathrm{P}$ maps $c_{K_{k}}^{r, \alpha+1 / k}(\mathbf{R})$ continuously into $\mathrm{c}^{r, \alpha+1 / k}(\mathbb{T})$ (considered as the space of periodic functions with period 1). The proof of surjectivity is the same as in the well-known $C^{\infty}$ case. Namely, take a compactly supported smooth function $\psi$ with $\int_{\mathbf{R}} \psi(x) \mathrm{d} x=1$ and define the function $\phi=\psi * \chi[0,1)$. Then, $\phi$ is smooth, compactly supported, and $P \phi=1$. From this, one easily obtains that $P(\phi f)=f$ for all $f \in \mathrm{c}^{r, \alpha+1 / k}(\mathbb{T})$. Surjectivity follows since $\phi f \in c_{K_{k}}^{r, \alpha+1 / k}(\mathbf{R})$ for all $k \in \mathbf{N}$ large enough. This concludes the proof of Theorem 15 .

Remark 19 Another strategy for (dis)proving nuclearity, which the authors have not pursued, would be to use the $\varepsilon$-entropy of compact sets and Mityagin's condition (see [20]). It should be sufficient to use the entropy numbers of embeddings of Besov spaces (see [26, Theorem 1.97.]).

\section{Examples of Hölder distributions}

In this section, we give several examples of Hölder distributions. The first example has already been mentioned in Introduction:

Example 20 Let us consider the distribution vp. $\left(\frac{1}{x}\right)$ on $\mathbf{R}$ whose action on $\varphi \in C_{c}^{\infty}(\mathbf{R})$ can be defined equivalently by

$$
\left\langle\operatorname{vp} \cdot\left(\frac{1}{\mathrm{x}}\right), \varphi\right\rangle=\lim _{\varepsilon \rightarrow 0+} \int_{\mathbf{R} \backslash[-\varepsilon, \varepsilon]} \frac{\varphi(x)}{x} \mathrm{~d} x=\int_{0}^{+\infty} \frac{\varphi(x)-\varphi(-x)}{x} \mathrm{~d} x .
$$

It is an example of a distribution whose classical order is precisely 1 . We shall demonstrate by a simple calculation that it is actually a Hölder distribution of order at most $\alpha$ for any $0<\alpha<1$, and hence of order $0+$. Indeed, fix one arbitrary $\alpha \in\langle 0,1\rangle$ and take $\varphi \in \mathrm{C}_{c}^{\infty}(\mathbf{R})$. Denote $K=\operatorname{supp} \varphi$. Using the second integral in the above definition, we get

$$
\begin{aligned}
& \left|\left\langle\operatorname{vp} \cdot\left(\frac{1}{\mathrm{x}}\right), \varphi\right\rangle\right|=\left|\int_{0}^{+\infty} \frac{\varphi(x)-\varphi(-x)}{x} \mathrm{~d} x\right| \\
& \quad \leq \int_{0}^{1} \frac{|\varphi(x)-\varphi(-x)|}{x} \mathrm{~d} x+\int_{1}^{+\infty} \frac{|\varphi(x)-\varphi(-x)|}{x} \mathrm{~d} x .
\end{aligned}
$$

The second integral can be bounded by:

$$
\int_{1}^{+\infty} \frac{|\varphi(x)-\varphi(-x)|}{x} \mathrm{~d} x \leq 2 \max _{x \in K}|x \varphi(x)| \int_{1}^{+\infty} \frac{\mathrm{d} x}{x^{2}}=2 \max _{x \in K}|x \varphi(x)| \leq 2|K|\|\varphi\|_{\mathrm{L}^{\infty}(K)} .
$$

Concerning the first integral, we can write

$$
\begin{aligned}
\int_{0}^{1} \frac{|\varphi(x)-\varphi(-x)|}{x} \mathrm{~d} x & =2 \int_{0}^{1} \frac{|\varphi(x)-\varphi(-x)|}{|2 x|^{\alpha}|2 x|^{1-\alpha}} \mathrm{d} x \leq 2^{\alpha} \sup _{x, y \in K, x \neq y} \frac{|\varphi(x)-\varphi(y)|}{|x-y|^{\alpha}} \int_{0}^{1} \frac{\mathrm{d} x}{x^{1-\alpha}} \\
& \leq \frac{2^{\alpha}}{1-\alpha} \sup _{x, y \in K, x \neq y} \frac{|\varphi(x)-\varphi(y)|}{|x-y|^{\alpha}} .
\end{aligned}
$$

Taking $C_{K}=\max \left\{2|K|, \frac{2^{\alpha}}{1-\alpha}\right\}$, we just need to apply the part (b) of Proposition 12 to finish the proof. 
Example 21 For $\beta \in\langle 1,2\rangle$, consider a distribution Pf. $\frac{1}{x_{+}^{\beta}}$ whose action on $\varphi \in \mathrm{C}_{c}^{\infty}(\mathbf{R})$ can be defined equivalently by

$$
\left\langle\operatorname{Pf} \cdot \frac{1}{x_{+}^{\beta}}, \varphi\right\rangle=\lim _{\varepsilon \rightarrow 0+}\left(\int_{\varepsilon}^{+\infty} \frac{\varphi(x)}{x^{\beta}} \mathrm{d} x-\frac{2 \varphi(0)}{\varepsilon^{\beta-1}}\right)=\int_{0}^{+\infty} \frac{\varphi(x)-\varphi(0)}{x^{\beta}} \mathrm{d} x .
$$

Its classical order is equal to 1 , yet we will show that it is a Hölder distribution of order at most $\alpha$ for any $\alpha \in\langle\beta-1,1\rangle$, and hence of order at most $(\beta-1)+$. Similarly as in the above example, we have

$$
\begin{aligned}
\left|\left\langle\operatorname{Pf} \cdot \frac{1}{\mathrm{x}_{+}^{\mathrm{fi}}}, \varphi\right\rangle\right| & =\left|\int_{0}^{+\infty} \frac{\varphi(x)-\varphi(0)}{x^{\beta}} \mathrm{d} x\right|=\int_{0}^{1} \frac{|\varphi(x)-\varphi(0)|}{x^{\beta}} \mathrm{d} x+\int_{1}^{+\infty} \frac{|\varphi(x)-\varphi(0)|}{x^{\beta}} \mathrm{d} x \\
& \leq \sup _{x, y \in K, x \neq y} \frac{|\varphi(x)-\varphi(y)|}{|x-y|^{\alpha}} \int_{0}^{1} \frac{\mathrm{d} x}{x^{\beta-\alpha}}+2|K|\|\varphi\|_{L^{\infty}(K)} .
\end{aligned}
$$

Since $\beta-\alpha<1$, the integral in the last line is finite and is equal to $\frac{1}{1-\beta+\alpha}$.

Example 22 Finite part (partie finie) of $\frac{1}{x^{2}}$, defined for $\varphi \in C_{c}^{\infty}(\Omega)$ by

$$
\left\langle\text { Pf. } \frac{1}{x^{2}}, \varphi\right\rangle=\lim _{\varepsilon \rightarrow 0+}\left(\int_{-\infty}^{-\varepsilon} \frac{\varphi(x)}{x^{2}} \mathrm{~d} x+\int_{\varepsilon}^{+\infty} \frac{\varphi(x)}{x^{2}} \mathrm{~d} x-\frac{2 \varphi(0)}{\varepsilon}\right)
$$

is a distribution of order at most $1+\alpha$ for every $\alpha \in\langle 0,1\rangle$ and hence of order $1+$. Indeed, this follows easily once we remark that Pf. $\frac{1}{\mathrm{x}^{2}}=-\left(\mathrm{vp} .\left(\frac{1}{\mathrm{x}}\right)\right)^{\prime}$. Its classical order is 2 .

\section{Fourier-analytic properties of distributions of real order and their test functions}

In this section, we use classical results to obtain some further properties of the introduced distributions of positive real order and their test functions. We start from a result in Sect. 4. There we obtained in Lemma 17 that the Fourier basis constitutes a Schauder basis for $C^{r, \alpha+}(\mathbb{T})$, a result which does not hold for $C^{r, \alpha}(\mathbb{T})$. We have the same result for the general $d$-dimensional case:

Theorem 23 The sequence $\left(\mathbf{x} \mapsto e^{2 \pi i \mathbf{k} \cdot \mathbf{x}}\right)_{\mathbf{k} \in \mathbf{Z}^{d}}$ is a Schauder basis for $\mathrm{C}^{r, \alpha+}\left(\mathbb{T}^{d}\right), r \in \mathbf{N}_{0}$, $0 \leq \alpha<1$. In fact,

$$
\lim _{N \rightarrow \infty} \sup _{\|f\|_{\mathrm{c}^{r, \alpha+1 / n}\left(\mathbb{T}^{d}\right)} \leq 1}\left\|S_{N} f-f\right\|_{\mathrm{c}^{r, \alpha+1 / m}\left(\mathbb{T}^{d}\right)}=0
$$

for all $m>n \gg 1$. Here $S_{N}$ denotes the (cubic) Fourier partial sum operator.

The proof is the same (up to obvious modifications), though we need a substitute for Lemma 16 (sometimes referred to as Jackson's theorem), which can be found in [2].

Some other classical results we are interested in are [16, Theorem 7.3.1., Theorem 7.6.6., and Theorem 7.9.3.]. We have the following consequence of [16, Lemma 7.9.2, Theorem 7.9.3.] and the inclusion [16, (7.9.6)]

Theorem $24 C_{c}^{\left[d\left(\frac{1}{p}-\frac{1}{2}\right)\right],\left(d\left(\frac{1}{p}-\frac{1}{2}\right)-\left[d\left(\frac{1}{p}-\frac{1}{2}\right)\right]\right)+}\left(\mathbf{R}^{d}\right), 1 \leq p<2$, is continuously embedded into the space of functions having Fourier transform in $L^{p}\left(\mathbf{R}^{d}\right)$, and each distribution having Fourier transform in $L^{q}\left(\mathbf{R}^{d}\right), q=1 /(1-1 / p) \in\langle 2, \infty\rangle$, is of order at most $d\left(\frac{1}{p}-\frac{1}{2}\right)+$. 
The fact that this is essentially the best result possible is contained in Theorem 7.6.6. in [16]. Namely, there one obtains by scaling that the classical order in general cannot be less than $d\left(\frac{1}{p}-\frac{1}{2}\right)$. The same is also true for our real order, and one easily sees that the same proof can be applied as in [16] as soon as one notices that for $0<\alpha<1, t \gg 1, \xi \in \mathbf{R}^{d}$ in a fixed bounded set, and $f$ smooth

$$
\sup _{\mathbf{h} \neq 0} \frac{\left|e^{i t f(\xi+\mathbf{h})}-e^{i t f(\xi)}\right|}{|\mathbf{h}|^{\alpha}} \leq C(f) t^{\alpha} .
$$

This inequality is applied to the expression [16, (7.6.12)] when calculating the Hölder norms of derivatives of the test functions $u_{t}=\widehat{u} e^{i t|\xi|^{2}}$ considered in the proof.

We also have the following extension (of one part) of the Paley-Wiener-Schwartz theorem.

Theorem 25 Let $K \subset \mathbf{R}^{d}$ be a open ball of radius $R$ and centre 0 . If $u$ is a Hölder distribution with order $\alpha \geq 0$ and with support contained in $K$, then

$$
|U(\zeta)| \lesssim(1+\|\zeta\|)^{\alpha} e^{2 \pi R\|\operatorname{Im} \zeta\|}
$$

where $\zeta \in \mathbf{C}^{d}$ and $U$ is the Fourier-Laplace transform of $u$.

We omit the proof since it is again a straightforward modification of the first part of the proof of [16, Theorem 7.3.1.]. One again needs inequality (2), and it is essentially the only additional ingredient needed to prove Theorem 25.

As is well known, the reverse of Theorem 25 does not hold. The simplest example is if we take the distribution $\chi_{[-1,1]}$ pv. $\frac{1}{x}$, then its Fourier-Laplace transform is up to a constant the complex sine integral function Si which satisfies (3) for $\alpha=0$. On the other hand, we know that $\chi_{[-1,1]} \mathrm{pv} \cdot \frac{1}{x}$ is not of order 0 , but $0+$. Actually, Theorem 24 hints that in general the gap can be much worse (up to $d / 2$ ).

In the remainder of this section, we give an example of a completely different proof of sharpness of Theorem 24 for the case $d=1$ and $p=1$. It is based on a concrete example and functional-analytic arguments.

We begin by giving an example of a compactly supported Hölder continuous function of degree $1 / 2$ on the real line which does not have an absolutely integrable Fourier transform. Our plan is to use a modification of a well-known example of a periodic Hölder continuous function whose Fourier series does not converge absolutely. This function is defined by the conditionally convergent series

$$
f(x)=\sum_{k=2}^{\infty} \frac{e^{i k \ln k}}{k} e^{2 \pi i k x} .
$$

The proof of the fact that this converges (and in fact uniformly) and of the fact that $f$ is Hölder continuous of order $1 / 2$ can be found in [27, Chapter VI, Theorem 3.1., p. 240] (see also [27, Chapter V, Theorem 4.2., p. 197]). There are two main steps in this proof. The first step (and the more difficult one) is to prove the estimate

$$
\sup _{x \in \mathbf{R}}\left|\sum_{k=2}^{N} e^{i k \ln k} e^{2 \pi i k x}\right| \leq C \sqrt{N}
$$

by using the van der Corput lemma. In the second step, one proves that the series converges (uniformly) to a Hölder continuous function of order $1 / 2$ using the above estimate and summation by parts. Now one can modify this example by considering it as a function on the 
real line and using an appropriate cutoff function (though it seems not any). The point of the following lemma is not the statement itself but rather the way we transfer example (4) from $\mathbb{T}$ to the real line.

Lemma 26 There exists a $C_{c}^{0, \frac{1}{2}}(\mathbf{R})$ function which is not in the Wiener algebra, i.e., it does not have an absolutely integrable Fourier transform.

Proof Denote $S_{n}(x)=\sum_{k=2}^{n} \frac{e^{i k \ln k}}{k} e^{2 \pi i k x}$. We have $S_{n} \rightarrow f$ uniformly and hence also in $\mathcal{S}^{\prime}(\mathbf{R})$. On the other hand, we know

$$
\mathcal{F} S_{n}(\xi)=\sum_{k=2}^{n} \frac{e^{i k \ln k}}{k} \delta_{k}(\xi),
$$

and since $\mathcal{F} S_{n} \rightarrow \mathcal{F} f$ in $\mathcal{S}^{\prime}(\mathbf{R})$, we have

$$
\mathcal{F} f(\xi)=\sum_{k=2}^{\infty} \frac{e^{i k \ln k}}{k} \delta_{k}(\xi),
$$

of course in $\mathcal{S}^{\prime}(\mathbf{R})$.

Take an arbitrary smooth function $\tilde{\psi}$ with compact support and $\mathcal{F} \tilde{\psi}(0)=1 / 2$. Define

$$
\psi=\tilde{\psi} * \chi_{[-1,1]},
$$

where $\chi_{[-1,1]}$ is the characteristic function of the set $[-1,1]$. Then, $\psi$ is again a compactly supported smooth function with Fourier transform

$$
\mathcal{F} \psi(\xi)=\mathcal{F} \tilde{\psi}(\xi) \mathcal{F} \chi_{[-1,1]}(\xi)=\mathcal{F} \tilde{\psi}(\xi) \frac{\sin (2 \pi \xi)}{\pi \xi} .
$$

The two main properties of the Fourier transform of $\psi$ we are going to use are that it is a Schwartz function and also that it has a zero at each integer, excluding the point $\xi=0$ where it is equal to 1 .

Now consider the function $g(x)=f(x) \psi(x)$. It is Hölder continuous of order $1 / 2$ and has compact support, so it only remains to prove that its Fourier transform is not absolutely integrable. We have

$$
\begin{aligned}
\mathcal{F} g(\xi) & =\overline{\mathcal{F}} f * \overline{\mathcal{F}} \psi(\xi)=\left\langle\overline{\mathcal{F}} f, \tau_{\xi} \mathcal{F} \psi\right\rangle=\left\langle\overline{\mathcal{F}} \lim _{n} S_{n}, \tau_{\xi} \mathcal{F} \psi\right\rangle \\
& =\lim _{n} \sum_{k=2}^{n} \frac{e^{i k \ln k}}{k}\left\langle\overline{\mathcal{F}} e^{2 \pi i k x}, \tau_{\xi} \mathcal{F} \psi\right\rangle \\
& =\lim _{n} \sum_{k=2}^{n} \frac{e^{i k \ln k}}{k}\left\langle\delta_{-k}, \tau_{\xi} \mathcal{F} \psi\right\rangle \\
& =\lim _{n} \sum_{k=2}^{n} \frac{e^{i k \ln k}}{k} \tau_{\xi} \mathcal{F} \psi(-k) \\
& =\sum_{k=2}^{\infty} \frac{e^{i k \ln k}}{k} \mathcal{F} \psi(-k-\xi),
\end{aligned}
$$

where in the fourth equality we used the fact that $S_{n} \rightarrow f$ in $\mathcal{S}^{\prime}(\mathbf{R})$. First, one should notice that $\mathcal{F} g(-l)=e^{i l \ln l} / l$ for $l>1$ an integer, and so $|\mathcal{F} g(-l)|=\left|e^{i l \ln l} / l\right|=1 / l$ (here we 
used that $\mathcal{F} \psi(l)=0$ for $l \neq 0$ ). To prove that $\mathcal{F} g$ is not absolutely integrable, it is sufficient to show that there exists a $\delta \in\langle 0,1 / 2\rangle$ such that

$$
|\mathcal{F} g(-l+\xi)|>\frac{1}{2}|\mathcal{F} g(-l)|
$$

for each $l>1$ an integer and all $|\xi|<\delta$ (the point is that the choice of $\delta$ is independent of l). From this, it would follow that

$$
\int_{\mathbf{R}}|g(\xi)| \mathrm{d} \xi \geq \sum_{l=2}^{\infty} \int_{|\xi|<\delta}|\mathcal{F} g(-l+\xi)| \mathrm{d} \xi \geq \frac{1}{2} \sum_{l=2}^{\infty} \int_{|\xi|<\delta}|\mathcal{F} g(-l)| \mathrm{d} \xi \geq \delta \sum_{l=2}^{\infty} \frac{1}{l},
$$

where the right-hand side diverges.

To obtain the desired $\delta$, one should notice that it is enough to prove that $(\mathcal{F} g)^{\prime}$ is $\mathcal{O}\left(\frac{1}{|\xi|}\right)$ as $|\xi| \rightarrow+\infty$, i.e., there exists a constant $A>0$ such that $\left|(\mathcal{F} g)^{\prime}\right| \leq \frac{A}{|\xi|}$ for $|\xi|>1$. Then, we could take $\delta=\min \{1 /(4 A), 1 / 4\}$ since by the mean value theorem there is a $\tilde{\xi}$ between $-l$ and $-l+\xi$ such that

$$
\begin{aligned}
& |\mathcal{F} g(-l+\xi)-\mathcal{F} g(-l)|=\left|(\mathcal{F} g)^{\prime}(-l+\tilde{\xi})\right||\xi| \\
& \quad \leq \frac{A|\xi|}{|-l+\tilde{\xi}|} \leq \frac{1 / 4}{l-1 / 4}=\frac{1}{4 l-1}<\frac{1}{2 l}=\frac{1}{2}|\mathcal{F} g(-l)|,
\end{aligned}
$$

and so we would get the desired inequality

$$
\begin{aligned}
\frac{1}{2}|\mathcal{F} g(-l)| & \leq \frac{1}{2}|\mathcal{F} g(-l)-\mathcal{F} g(-l+\xi)|+\frac{1}{2}|\mathcal{F} g(-l+\xi)| \\
& <\frac{1}{4}|\mathcal{F} g(-l)|+\frac{1}{2}|\mathcal{F} g(-l+\xi)|,
\end{aligned}
$$

that is $|\mathcal{F} g(-l+\xi)|>\frac{1}{2}|\mathcal{F} g(-l)|$, for $|\xi|<\delta$.

Finally, we need to prove the decay property at infinity of $(\mathcal{F} g)^{\prime}$. Recall $\mathcal{F} g(\xi)=\overline{\mathcal{F}} f *$ $\overline{\mathcal{F}} \psi(\xi)$, so we have

$$
(\mathcal{F} g)^{\prime}(\xi)=\overline{\mathcal{F}} f *(\overline{\mathcal{F}} \psi)^{\prime}(\xi) .
$$

Then, by a similar calculation as before, one obtains

$$
(\mathcal{F} g)^{\prime}(\xi)=\sum_{k=2}^{\infty} \frac{e^{i k \ln k}}{k} \varphi(-k-\xi),
$$

where $\varphi$ is some Schwartz function. Since the above expression is a convolution between the functions of decay $1 / k$ and of arbitrary decay, the decay of the convolution is at best $\mathcal{O}\left(\frac{1}{|\xi|}\right)$. This concludes the proof of the lemma.

In particular, now we know that the space of $C_{c}^{0,1 / 2}(\mathbf{R})$ is not a subset of the Wiener algebra, and therefore, also $\mathrm{c}_{c}^{0,1 / 2-\varepsilon}(\mathbf{R})$ is not a subset of the Wiener algebra for any $0<\varepsilon<1 / 2$. But from this it is not immediately clear that a distribution satisfying

$$
|\langle T, \varphi\rangle| \leq C\|\mathcal{F} \varphi\|_{\mathrm{L}^{1}(\mathbf{R})}
$$

is not generally of order at most $1 / 2$. We prove that there exist distributions satisfying the above bound, but which are of order at least $1 / 2-\varepsilon$ for $\varepsilon$ arbitrarily small. For convenience, denote by $X$ the Wiener algebra (a Banach space with norm $\|x\|_{X}=\|\mathcal{F} x\|_{L^{1}(\mathbf{R})}$, and hence, its dual $X^{\prime}$ is the space of distributions with Fourier transform in $L^{\infty}(\mathbf{R})$, the uniform norm 
being $\left.\|T\|_{X^{\prime}}=\|\mathcal{F} T\|_{L^{\infty}(\mathbf{R})}, T \in X^{\prime}\right)$ and by $Y$ the space $c_{c}^{0,1 / 2-\varepsilon}(\mathbf{R})$, a strict inductive limit of Banach spaces $Y_{k}=\mathrm{c}_{K_{k}}^{0,1 / 2-\varepsilon}(\mathbf{R})$, for a fixed small $\varepsilon$. Recall also that $Y^{\prime}$ is a Fréchet space with the strong topology. We need the following simple lemma.

Lemma 27 Assume that each distribution which can be extended to an element of $X^{\prime}$ can also be extended to an element of $Y^{\prime}$. Also assume that the induced embedding of $X^{\prime} \rightarrow Y^{\prime}$ is continuous in the pair of topologies (uniform topology on $X^{\prime}$, weak* topology on $Y^{\prime}$ ). Then, $Y$ embeds continuously into $X$.

Proof First, we have to prove that the extensions are compatible, i.e., the actions coincide on the set $X \cap Y$. By continuity, it is enough to construct for each $y \in X \cap Y$ a sequence in $\mathrm{C}_{c}^{\infty}(\mathbf{R})$ which converges in $X$ and $Y$ topologies at the same time. This is easily done by using the standard mollifier.

Now by assumption, $X^{\prime}$ embeds into $Y^{\prime}$, and as the restriction operator maps $Y^{\prime}$ to $Y_{k}^{\prime}$, we can ask whether a bound of the form $\|f\|_{Y_{k}^{\prime}} \leq C_{k}\|f\|_{X^{\prime}}$ holds. This indeed holds by the following argument. Consider the bilinear form on $X^{\prime} \times Y_{k}$ defined by $(f, y) \mapsto\langle f, y\rangle=$ $f(y)$. This form satisfies the bound $|\langle f, y\rangle| \leq C_{y}\|f\|_{X^{\prime}}$ by continuity of the inclusion $X^{\prime} \rightarrow Y^{\prime}$ and the bound $|\langle f, y\rangle| \leq C_{f}\|y\|_{Y_{k}^{\prime}}$ by continuity of $f$ on $Y_{k}^{\prime}$. By the uniform boundedness principle, we have $|\langle f, y\rangle| \leq C_{k}\|y\|_{Y_{k}^{\prime}}\|f\|_{X^{\prime}}$.

Finally, one should notice that for $y \in Y_{k}$ we have

$$
\|y\|_{X}=\sup _{\substack{f \in \Sigma \\\|f\|_{X^{\prime}} \leq 1}}|\langle f, y\rangle| \leq \sup _{\substack{f \in \Sigma \\\|f\|_{Y_{k}^{\prime}} \leq C_{k}}}|\langle f, y\rangle| \leq \sup _{\substack{f \in \Sigma \\\|f\|_{Y_{k}^{\prime}} \leq C_{k}}}\|f\|_{Y_{k}^{\prime}}\|y\|_{Y_{k}} \leq C_{k}\|y\|_{Y_{k}} .
$$

Here $\Sigma$ denotes the subspace of $X^{\prime}$ of all distributions whose Fourier transform are simple functions on $\mathbf{R}$ vanishing outside a set of finite measure, and so as the Fourier transform of $y$ is a $C_{0}(\mathbf{R})$ function as $y$ is integrable, the (first) equality is justified by the converse of Hölder inequality. Therefore, we conclude that $Y_{k}$ embeds continuously into $X$ for each $k$ in the pair of norm topologies, and so $Y$ also embeds continuously into $X$.

Remark 28 One could circumvent using the converse Hölder inequality if $X$ were reflexive, but this is not the case since it is isomorphic to $L^{1}$ as a Banach space. On the other hand, a proof using density should work. Indeed, if we additionally assume $y \in X$, we have

$$
\|y\|_{X}=\sup _{\substack{f \in X^{\prime} \\\|f\|_{X^{\prime}} \leq 1}}|\langle f, y\rangle|
$$

and so we have an embedding of $X \cap Y_{k}$ into $X$ in the pair of norm topologies of $Y_{k}$ and $X$. It remains to prove that $X \cap Y_{k}$ is dense in $Y_{k}$ (this is not that simple; it is sufficient to prove that $C_{K_{k}}^{\infty}$ is dense in $Y_{k}$, but since mollifying will push the support outside $K_{k}$, one needs to use a cutoff function and analyse what is happening at the boundary, and for this, a usual strategy for higher dimensions is first to straighten the boundary for which one needs to assume some smoothness on $\partial K_{k}$ ). In trying to circumvent even a density argument, one could conclude that $X \cap Y \rightarrow X$ is continuous in the pair of the inductive limit topology of the sequence $\left(X \cap Y_{k}\right)_{k}$ and the norm topology of $X$. Unfortunately, the inductive limit topology of the sequence $\left(X \cap Y_{k}\right)_{k}$ on $X \cap Y$ does not in general coincide with the subspace topology on $X \cap Y$ inherited from $Y$. In fact, in general it can be strictly finer than the subspace topology when considering strict inductive limits of Fréchet spaces even if the subspace is closed (see [25, Remark 13.2.]). One should compare this with Theorem 3 e). 
Theorem 29 The dual of the Wiener algebra does not embed into $\mathcal{D}_{1 / 2-\varepsilon}(\mathbf{R})$ for any $\varepsilon$, where both spaces are considered as subsets of the space of distributions.

Proof Now since we know that $Y=c_{c}^{0,1 / 2-\varepsilon}(\mathbf{R})$ does not embed into the Wiener algebra $X$, by the previous lemma, it follows that either $X^{\prime}$ is not a subset of $Y^{\prime}$, or that the embedding $X^{\prime} \rightarrow Y^{\prime}$ is not continuous in the pair of norm and weak* topology. We argue that the latter case is not possible. Assume that we have the (not necessarily continuous) embedding $X^{\prime} \rightarrow Y^{\prime}$. Its graph is closed in the pair of strong topologies, since both $X^{\prime}$ and $Y^{\prime}$ with strong topologies embed continuously into the space of distributions (with say the weak* topology). But since both $X^{\prime}$ and $Y^{\prime}$ are Fréchet in these topologies, the closed graph theorem is valid and we obtain an even stronger continuity than needed.

We conclude that there exists a distribution satisfying

$$
|\langle T, \varphi\rangle| \leq C\|\mathcal{F} \varphi\|_{\mathrm{L}^{1}(\mathbf{R})}
$$

which is not of order at most $1 / 2-\varepsilon$ and hence has order strictly larger than $1 / 2-\varepsilon$. Therefore, we see that we cannot lower the order below $\frac{1}{2}$. We could say that a distribution on the real line having an a.e. bounded Fourier transform is of order somewhere between “ $\frac{1}{2}-$ " and $\frac{1}{2}+$.

Of course, it would be of interest to provide examples for greater dimensions. This would likely entail an appropriate modification of (4) and proving the associated estimates.

\section{Convolution of Hölder distributions}

Let $u \in \mathcal{D}_{r+\alpha}^{\prime}\left(\mathbf{R}^{d}\right)$ and $v \in \mathcal{D}_{q+\beta}^{\prime}\left(\mathbf{R}^{d}\right)$, where $r, q \in \mathbf{N}_{0}$ and $\alpha, \beta \in[0,1\rangle$, and assume the following compatibility condition on their supports $K_{u}$ and $K_{v}$ :

For any compact set $K \subset \mathbf{R}^{d}$, there exist compact sets $K_{1}, K_{2} \subset \mathbf{R}^{d}$ such that

$$
\left(\mathbf{x} \in K_{u}, \mathbf{y} \in K_{v} \wedge \mathbf{x}+\mathbf{y} \in K\right) \Longrightarrow \mathbf{x} \in K_{1}, \quad \mathbf{y} \in K_{2} .
$$

Since $u$ and $v$ are distributions, it is standard to define their convolution $u * v \in \mathcal{D}^{\prime}\left(\mathbf{R}^{d}\right)$ by its action on a test function $\varphi \in C_{K}^{\infty}\left(\mathbf{R}^{d}\right)$ (with obvious abuse of notation)

$$
\langle u * v, \varphi\rangle=\left\langle u(\mathbf{x}) \otimes v(\mathbf{y}), \rho_{1}(\mathbf{x}) \rho_{2}(\mathbf{y}) \varphi(\mathbf{x}+\mathbf{y})\right\rangle,
$$

where $\rho_{1} \in C_{c}^{\infty}\left(\mathbf{R}^{d}\right)$ is equal to one on a neighborhood of $K_{1}$ and $\rho_{2} \in C_{c}^{\infty}\left(\mathbf{R}^{d}\right)$ is equal to one on a neighborhood of $K_{2}$. The definition does not depend on the choice of cutoff functions $\rho_{1}$ and $\rho_{2}$, and furthermore, the following formula holds:

$$
\langle u * v, \varphi\rangle=\langle v(\mathbf{y}),\langle u(\mathbf{x}), \varphi(\mathbf{x}+\mathbf{y})\rangle\rangle=\langle u(\mathbf{x}),\langle v(\mathbf{y}), \varphi(\mathbf{x}+\mathbf{y})\rangle\rangle .
$$

We will show that $u * v$ is in fact a Hölder distribution of order at most $r+q+\alpha+\beta$. Recall that for $\alpha, \beta \geq 0$ we denote by $[\alpha+\beta]$ the greatest integer less than $\alpha+\beta$ and by $\{\alpha+\beta\}$ the difference $\alpha+\beta-[\alpha+\beta] \in[0,1\rangle$.

Let us first show the following result.

Lemma 30 Let $\Omega_{1} \subset \mathbf{R}^{d_{1}}$ and $\Omega_{2} \subset \mathbf{R}^{d_{2}}$ be open subsets and take $r, q \in \mathbf{N}_{0}$ and $\alpha, \beta \in$ $[0,1\rangle$. Assume that $\varphi \in \mathrm{c}^{r+q+[\alpha+\beta],\{\alpha+\beta\}}\left(\Omega_{1} \times \Omega_{2}\right)$ satisfies:

$$
\begin{aligned}
& \left(\forall \mathbf{y}^{\prime} \in \Omega_{2}\right)\left(\exists U\left(\mathbf{y}^{\prime}\right) \subset \Omega_{2} \text { neighborhood of } \mathbf{y}^{\prime}\right)\left(\exists K\left(\mathbf{y}^{\prime}\right) \subset \Omega_{1} \text { compact }\right): \\
& \quad \operatorname{supp}(\varphi(., \mathbf{y})) \subset K\left(\mathbf{y}^{\prime}\right), \quad \forall \mathbf{y} \in U\left(\mathbf{y}^{\prime}\right) .
\end{aligned}
$$


Then for any $u \in \mathcal{D}_{r+\alpha}^{\prime}\left(\Omega_{1}\right)$, one has:

$$
\mathbf{y} \mapsto\langle u(\mathbf{x}), \varphi(\mathbf{x}, \mathbf{y})\rangle \in \mathrm{c}^{q, \beta}\left(\Omega_{2}\right) .
$$

In particular, if $\varphi$ is infinitely differentiable, then the same is true for

$$
\mathbf{y} \mapsto\langle u(\mathbf{x}), \varphi(\mathbf{x}, \mathbf{y})\rangle .
$$

Proof Notice that for every fixed $\mathbf{y} \in \Omega_{2}$, function $\mathbf{x} \mapsto \varphi(\mathbf{x}, \mathbf{y})$ belongs to $c_{c}^{r, \alpha}\left(\Omega_{1}\right)$. Thus, the function

$$
\Phi(\mathbf{y})=\langle u(\mathbf{x}), \varphi(\mathbf{x}, \mathbf{y})\rangle
$$

is well defined. It remains to show that $\Phi$ belongs to the space $c^{q, \beta}\left(\Omega_{2}\right)$. Let us first prove continuity. For $\mathbf{h}$ such that $|\mathbf{h}|<\delta$, define

$$
\varphi_{\mathbf{h}}(\mathbf{x}, \mathbf{y})=\varphi(\mathbf{x}, \mathbf{y}+\mathbf{h})-\varphi(\mathbf{x}, \mathbf{y}),
$$

and write

$$
\Phi(\mathbf{y}+\mathbf{h})-\Phi(\mathbf{y})=\left\langle u(\mathbf{x}), \varphi_{\mathbf{h}}(\mathbf{x}, \mathbf{y})\right\rangle .
$$

Note that if we take $\delta$ small enough, then $\varphi_{\mathbf{h}}(\mathbf{x}, \mathbf{y})$ have supports contained in a fixed compact set independent of $\mathbf{h}$. In order to show that $\Phi$ is continuous, it is enough to show that $\varphi_{\mathbf{h}}(., \mathbf{y}) \rightarrow 0$ in $c_{c}^{r, \alpha}\left(\Omega_{1}\right)$ as $|\mathbf{h}| \rightarrow 0$. This immediately follows from

$$
\left|\partial^{\gamma} \varphi_{\mathbf{h}}(\mathbf{x}, \mathbf{y})\right|=\left|\partial^{\gamma} \varphi(\mathbf{x}, \mathbf{y}+\mathbf{h})-\partial^{\gamma} \varphi(\mathbf{x}, \mathbf{y})\right| \leq C_{U(\mathbf{y})}^{\gamma}(\mathbf{h})|\mathbf{h}|^{\alpha},
$$

where by $C_{U(\mathbf{y})}^{\gamma}(\mathbf{h})$ we have denoted the Hölder seminorm of the function $\partial^{\gamma} \varphi$ with respect to the second variable on the set $U(\mathbf{y})$, and by $\boldsymbol{\gamma}$ a multi-index such that $|\boldsymbol{\gamma}| \leq r$. It remains to recall that $C_{U(\mathbf{y})}^{\gamma}(\mathbf{h}) \rightarrow 0$ as $|\mathbf{h}| \rightarrow 0$.

Next we prove that $\Phi$ is a $q$ times differentiable function and that we have the formula

$$
\partial^{\gamma} \Phi(\mathbf{y})=\left\langle u(\mathbf{x}), \partial_{\mathbf{y}}^{\gamma} \varphi(\mathbf{x}, \mathbf{y})\right\rangle,
$$

for all $|\boldsymbol{\gamma}| \leq q$. Let us assume that $\Phi$ is $k-1<q$ times differentiable and that the formula is true for all $|\boldsymbol{\gamma}| \leq k-1$. We consider the function (for $h \in\langle 0, \delta\rangle$ )

$$
\theta_{h}(\mathbf{x}, \mathbf{y})=\frac{\partial^{\gamma} \varphi\left(\mathbf{x}, \mathbf{y}+h e_{j}\right)-\partial^{\gamma} \varphi(\mathbf{x}, \mathbf{y})}{h}-\partial_{y_{j}} \partial^{\gamma} \varphi(\mathbf{x}, \mathbf{y}),
$$

where $e_{j}$ is a vector of the standard basis of $\mathbf{R}^{d_{2}}$, and write

$$
\frac{\partial^{\gamma} \Phi\left(\mathbf{y}+h e_{j}\right)-\partial^{\gamma} \Phi(\mathbf{y})}{h}-\left\langle u(\mathbf{x}), \partial_{y_{j}} \partial^{\gamma} \varphi(\mathbf{x}, \mathbf{y})\right\rangle=\left\langle u(\mathbf{x}), \theta_{h}(\mathbf{x}, \mathbf{y})\right\rangle .
$$

To show that $\Phi$ is of class $C^{k}$, it would be enough to show that $\theta_{h}(., \mathbf{y}) \rightarrow 0$ in $c_{c}^{r, \alpha}\left(\Omega_{1}\right)$ as $h \rightarrow 0$. This can be done in precisely the same way as it was done for $\varphi_{\mathbf{h}}$ above after one uses the mean value theorem to rewrite $\theta_{h}$ as

$$
\theta_{h}(\mathbf{x}, \mathbf{y})=\partial_{y_{j}} \partial^{\gamma} \varphi\left(\mathbf{x}, \mathbf{y}+\tilde{h}(\mathbf{x}, \mathbf{y}) e_{j}\right)-\partial_{y_{j}} \partial^{\gamma} \varphi(\mathbf{x}, \mathbf{y}),
$$

where $|\tilde{h}(\mathbf{x}, \mathbf{y})| \leq h$.

The final step is to prove that partial derivatives of order $q$ are Hölder of order $\beta$. We can take $\beta>0$ as the case $\beta=0$ is actually already proven. Take an arbitrary $\mathbf{y}^{\prime} \in \Omega_{2}$, 
and $\delta>0$ such that an open ball of radius $\delta$ centered around $\mathbf{y}^{\prime}$ is contained in $U\left(\mathbf{y}^{\prime}\right)$. For $\mathbf{y}, \hat{\mathbf{y}} \in K\left(\mathbf{y}^{\prime}, \delta\right) \subset U\left(\mathbf{y}^{\prime}\right)$, and $|\boldsymbol{\gamma}|=q$, we write:

$$
\begin{aligned}
& \frac{\left|\partial^{\gamma} \Phi(\mathbf{y})-\partial^{\gamma} \Phi(\hat{\mathbf{y}})\right|}{|\mathbf{y}-\hat{\mathbf{y}}|^{\beta}}=\left|\left\langle u(\mathbf{x}), \frac{\partial^{\gamma} \varphi(\mathbf{x}, \mathbf{y})-\partial^{\gamma} \varphi(\mathbf{x}, \hat{\mathbf{y}})}{|\mathbf{y}-\hat{\mathbf{y}}|^{\beta}}\right\rangle\right| \\
& \quad \lesssim \sum_{\left|\gamma_{1}\right| \leq r+q} \| \frac{\partial^{\gamma_{1}} \varphi(\mathbf{x}, \mathbf{y})-\partial^{\gamma_{1}} \varphi(\mathbf{x}, \hat{\mathbf{y}})}{|\mathbf{y}-\hat{\mathbf{y}}|^{\beta}}||_{L_{\mathbf{x}}^{\infty}} \\
& \quad+\sum_{\left|\gamma_{2}\right|=r+q} \sup _{\mathbf{x} \neq \hat{\mathbf{x}}} \frac{\left|\partial^{\gamma_{2}} \varphi(\mathbf{x}, \mathbf{y})-\partial^{\gamma_{2}} \varphi(\mathbf{x}, \hat{\mathbf{y}})-\partial^{\gamma_{2}} \varphi(\hat{\mathbf{x}}, \mathbf{y})+\partial^{\gamma_{2}} \varphi(\hat{\mathbf{x}}, \hat{\mathbf{y}})\right|}{|\mathbf{y}-\hat{\mathbf{y}}|^{\beta}|\mathbf{x}-\hat{\mathbf{x}}|^{\alpha}}
\end{aligned}
$$

Since $|\mathbf{y}-\hat{\mathbf{y}}|^{\beta}=|(\mathbf{x}, \mathbf{y})-(\mathbf{x}, \hat{\mathbf{y}})|^{\beta}$, the terms in the first sum in the above bound are bounded by the $\beta$-Hölder seminorms of the functions $\partial^{\gamma+\gamma_{1}} \varphi$. In fact, they go to 0 when $|\mathbf{y}-\hat{\mathbf{y}}| \rightarrow 0$ since $\varphi$ is in $\mathrm{c}_{c}^{r+q, \beta}\left(\Omega_{1} \times \Omega_{2}\right)$ and the variables go over a precompact set $K\left(\mathbf{y}^{\prime}\right) \times K(0, \delta)$, where $K\left(\mathbf{y}^{\prime}\right)$ is the compact set as in support condition (6).

For the second sum, we have to consider two cases separately. In the case when $\alpha+\beta<1$, using the inequality

$$
\frac{\min \left\{|\mathbf{x}-\hat{\mathbf{x}}|^{\alpha+\beta},|\mathbf{y}-\hat{\mathbf{y}}|^{\alpha+\beta}\right\}}{|\mathbf{x}-\hat{\mathbf{x}}|^{\alpha}|\mathbf{y}-\hat{\mathbf{y}}|^{\beta}} \leq 1,
$$

one gets

$$
\begin{aligned}
& \sup _{\mathbf{y} \neq \hat{\mathbf{y}} \mathbf{x} \neq \hat{\mathbf{x}}} \frac{\left|\partial^{\gamma_{2}} \varphi(\mathbf{x}, \mathbf{y})-\partial^{\gamma_{2}} \varphi(\mathbf{x}, \hat{\mathbf{y}})-\partial^{\gamma_{2}} \varphi(\hat{\mathbf{x}}, \mathbf{y})+\partial^{\gamma_{2}} \varphi(\hat{\mathbf{x}}, \hat{\mathbf{y}})\right|}{|\mathbf{y}-\hat{\mathbf{y}}|^{\beta}|\mathbf{x}-\hat{\mathbf{x}}|^{\alpha}} \\
& \quad \leq 2 \sup _{\mathbf{z} \neq \hat{\mathbf{z}}} \frac{\left|\partial^{\gamma_{2}} \varphi(\mathbf{z})-\partial^{\gamma_{2}} \varphi(\hat{\mathbf{z}})\right|}{|\mathbf{z}-\hat{\mathbf{z}}|^{\alpha+\beta}},
\end{aligned}
$$

where $\mathbf{y}, \hat{\mathbf{y}}$ go over $K\left(\mathbf{y}^{\prime}, \delta\right)$, and $\mathbf{z}, \hat{\mathbf{z}}$ go over $K\left(\mathbf{y}^{\prime}\right) \times K\left(\mathbf{y}^{\prime}, \delta\right)$. Thus, $\Phi \in C^{q, \beta}\left(\Omega_{2}\right)$. We can in fact show $\Phi \in c^{q, \beta}\left(\Omega_{2}\right)$. Namely, we have for any sufficiently small $\eta>0$

$$
\begin{aligned}
& \sup _{\substack{\mathbf{y} \neq \hat{\mathbf{y}} \\
|\mathbf{y}-\hat{\mathbf{y}}|<\eta}} \sup _{\substack{\mathbf{x} \neq \hat{\mathbf{x}}\\
}} \frac{\left|\partial^{\gamma_{2}} \varphi(\mathbf{x}, \mathbf{y})-\partial^{\gamma_{2}} \varphi(\mathbf{x}, \hat{\mathbf{y}})-\partial^{\gamma_{2}} \varphi(\hat{\mathbf{x}}, \mathbf{y})+\partial^{\gamma_{2}} \varphi(\hat{\mathbf{x}}, \hat{\mathbf{y}})\right|}{|\mathbf{y}-\hat{\mathbf{y}}|^{\beta}|\mathbf{x}-\hat{\mathbf{x}}|^{\alpha}} \\
& \leq 2 \sup _{\substack{\mathbf{z} \neq \hat{\mathbf{z}} \\
|\mathbf{z}-\hat{\mathbf{z}}|<\eta}} \frac{\left|\partial^{\gamma_{2}} \varphi(\mathbf{z})-\partial^{\gamma_{2}} \varphi(\hat{\mathbf{z}})\right|}{|\mathbf{z}-\hat{\mathbf{z}}|^{\alpha+\beta}},
\end{aligned}
$$

and so we get that the second sum in (7) also tends to 0 as $|\mathbf{y}-\hat{\mathbf{y}}| \rightarrow 0$.

In the case $\alpha+\beta \geq 1$, we need the inequalities

$$
\begin{aligned}
& \left|\partial^{\gamma_{2}} \varphi(\mathbf{x}, \mathbf{y})-\partial^{\boldsymbol{\gamma}_{2}} \varphi(\mathbf{x}, \hat{\mathbf{y}})-\partial^{\gamma_{2}} \varphi(\hat{\mathbf{x}}, \mathbf{y})+\partial^{\gamma_{2}} \varphi(\hat{\mathbf{x}}, \hat{\mathbf{y}})\right| \\
& \quad \lesssim|\mathbf{x}-\hat{\mathbf{x}}| \sup _{\tilde{\mathbf{x}} \in[\mathbf{x}, \hat{\mathbf{x}}]} \sup _{\left|\boldsymbol{\gamma}_{3}\right|=r+q+1}\left|\partial^{\gamma_{3}} \varphi(\tilde{\mathbf{x}}, \mathbf{y})-\partial^{\gamma_{3}} \varphi(\tilde{\mathbf{x}}, \hat{\mathbf{y}})\right| \\
& \left|\partial^{\gamma_{2}} \varphi(\mathbf{x}, \mathbf{y})-\partial^{\boldsymbol{\gamma}_{2}} \varphi(\mathbf{x}, \hat{\mathbf{y}})-\partial^{\gamma_{2}} \varphi(\hat{\mathbf{x}}, \mathbf{y})+\partial^{\gamma_{2}} \varphi(\hat{\mathbf{x}}, \hat{\mathbf{y}})\right| \\
& \quad \lesssim|\mathbf{y}-\hat{\mathbf{y}}| \sup _{\tilde{\mathbf{y}} \in[\mathbf{y}, \hat{\mathbf{y}}]} \sup _{\left|\gamma_{3}\right|=r+q+1}\left|\partial^{\gamma_{3}} \varphi(\mathbf{x}, \tilde{\mathbf{y}})-\partial^{\boldsymbol{\gamma}_{3}} \varphi(\hat{\mathbf{x}}, \tilde{\mathbf{y}})\right|
\end{aligned}
$$


obtained by applying the mean value theorem to functions $\mathbf{x} \mapsto \partial^{\gamma_{2}} \varphi(\mathbf{x}, \mathbf{y})-\partial^{\gamma_{2}} \varphi(\mathbf{x}, \hat{\mathbf{y}})$ and $\mathbf{y} \mapsto \partial^{\gamma_{2}} \varphi(\mathbf{x}, \mathbf{y})-\partial^{\gamma_{2}} \varphi(\hat{\mathbf{x}}, \mathbf{y})$. Here $[\mathbf{x}, \hat{\mathbf{x}}]$ (resp. $\left.[\mathbf{y}, \hat{\mathbf{y}}]\right)$ is the line segment connecting the points $\mathbf{x}$ and $\hat{\mathbf{x}}$ (resp. $\mathbf{y}$ and $\hat{\mathbf{y}}$ ). Therefore, if $M:=\min \{|\mathbf{x}-\hat{\mathbf{x}}|,|\mathbf{y}-\hat{\mathbf{y}}|\}$, then

$$
\begin{aligned}
& \left|\partial^{\gamma_{2}} \varphi(\mathbf{x}, \mathbf{y})-\partial^{\gamma_{2}} \varphi(\mathbf{x}, \hat{\mathbf{y}})-\partial^{\gamma_{2}} \varphi(\hat{\mathbf{x}}, \mathbf{y})+\partial^{\gamma_{2}} \varphi(\hat{\mathbf{x}}, \hat{\mathbf{y}})\right| \\
& \quad \lesssim M \sup _{|\mathbf{z}-\hat{\mathbf{z}}| \geq M\left|\boldsymbol{\gamma}_{3}\right|=r+q+1} \sup \left|\partial^{\gamma_{3}} \varphi(\mathbf{z})-\partial^{\boldsymbol{\gamma}_{3}} \varphi(\hat{\mathbf{z}})\right| .
\end{aligned}
$$

Now using inequalities (8) and (11), we get

$$
\begin{aligned}
& \sup _{\mathbf{y} \neq \hat{\mathbf{y}} \mathbf{x} \neq \hat{\mathbf{x}}} \frac{\left|\partial^{\gamma_{2}} \varphi(\mathbf{x}, \mathbf{y})-\partial^{\boldsymbol{\gamma}_{2}} \varphi(\mathbf{x}, \hat{\mathbf{y}})-\partial^{\gamma_{2}} \varphi(\hat{\mathbf{x}}, \mathbf{y})+\partial^{\boldsymbol{\gamma}_{2}} \varphi(\hat{\mathbf{x}}, \hat{\mathbf{y}})\right|}{|\mathbf{y}-\hat{\mathbf{y}}|^{\beta}|\mathbf{x}-\hat{\mathbf{x}}|^{\alpha}} \\
& \quad \lesssim \sup _{\left|\gamma_{3}\right|=r+q+1} \sup _{\mathbf{z} \neq \hat{\mathbf{z}}} \frac{\left|\partial^{\gamma{ }} \varphi(\mathbf{z})-\partial^{\boldsymbol{\gamma}_{3}} \varphi(\hat{\mathbf{z}})\right|}{|\mathbf{z}-\hat{\mathbf{z}}|^{\alpha+\beta-1}},
\end{aligned}
$$

where $\mathbf{z}, \hat{\mathbf{z}}$ go over $K\left(\mathbf{y}^{\prime}\right) \times K\left(\mathbf{y}^{\prime}, \delta\right)$. Thus, $\Phi \in C^{q, \beta}\left(\Omega_{2}\right)$. We can actually prove the needed stronger result $\Phi \in c^{q, \beta}\left(\Omega_{2}\right)$, though in a slightly different way than in the case $\alpha+\beta<1$. Namely, using the geometric mean of (9) with weight $(1-\beta) /(2-\alpha-\beta)$ and (10) with weight $(1-\alpha) /(2-\alpha-\beta)$, one easily gets the inequality

$$
\begin{aligned}
& \frac{\left|\partial^{\gamma_{2}} \varphi(\mathbf{x}, \mathbf{y})-\partial^{\gamma_{2}} \varphi(\mathbf{x}, \hat{\mathbf{y}})-\partial^{\gamma_{2}} \varphi(\hat{\mathbf{x}}, \mathbf{y})+\partial^{\gamma_{2}} \varphi(\hat{\mathbf{x}}, \hat{\mathbf{y}})\right|}{|\mathbf{y}-\hat{\mathbf{y}}|^{\beta}|\mathbf{x}-\hat{\mathbf{x}}|^{\alpha}} \\
& \lesssim \sup _{\left|\gamma_{3}\right|=r+q+1} \sup _{|\mathbf{z}-\hat{\mathbf{z}}|=|\mathbf{x}-\hat{\mathbf{x}}|}\left(\frac{\left|\partial^{\gamma} \varphi(\mathbf{z})-\partial^{\gamma_{3}} \varphi(\hat{\mathbf{z}})\right|}{|\mathbf{z}-\hat{\mathbf{z}}|^{\alpha+\beta-1}}\right)^{\frac{1-\alpha}{2-\alpha-\beta}} \\
& \quad \times \sup _{\left|\gamma_{3}\right|=r+q+1|\mathbf{z}-\hat{\mathbf{z}}|=|\mathbf{y}-\hat{\mathbf{y}}|}\left(\frac{\left|\partial^{\gamma_{3}} \varphi(\mathbf{z})-\partial^{\gamma_{3}} \varphi(\hat{\mathbf{z}})\right|}{|\mathbf{z}-\hat{\mathbf{z}}|^{\alpha+\beta-1}}\right)^{\frac{1-\beta}{2-\alpha-\beta}} .
\end{aligned}
$$

Our claim follows now easily since the second factor on the right-hand side of the previous inequality goes to 0 when $|\mathbf{y}-\hat{\mathbf{y}}| \rightarrow 0$ because $\varphi \in \mathrm{c}^{r+q+1, \alpha+\beta-1}\left(\Omega_{1} \times \Omega_{2}\right)$.

Remark 31 For $\zeta \in c_{c}^{r, \alpha}\left(\mathbf{R}^{d}\right)$, the function $\varphi: \mathbf{R}^{d} \times \mathbf{R}^{d} \rightarrow \mathbf{C}$ defined by $\varphi(\mathbf{x}, \mathbf{y})=\zeta(\mathbf{x}+\mathbf{y})$ satisfies the support condition assumed in the Lemma 30. It is clear that $\varphi \in c^{r, \alpha}\left(\mathbf{R}^{d} \times \mathbf{R}^{d}\right)$, and we can take $U\left(\mathbf{y}^{\prime}\right)$ to be the open unit ball around $\mathbf{y}^{\prime}$ and $K\left(\mathbf{y}^{\prime}\right)$ to be the set difference between supp $(\zeta)$ and the closure of $U\left(\mathbf{y}^{\prime}\right)$.

Theorem 32 Assume $u \in \mathcal{D}_{r+\alpha}^{\prime}\left(\mathbf{R}^{d}\right)$ and $v \in \mathcal{D}_{q+\beta}^{\prime}\left(\mathbf{R}^{d}\right)$ satisfy the compatibility condition on their supports (5). Then, $u * v \in \mathcal{D}_{r+q+\alpha+\beta}^{\prime}\left(\mathbf{R}^{d}\right)$.

Proof Take $\varphi \in C_{c}^{\infty}\left(\mathbf{R}^{d}\right)$. We already know that $u * v$ is a distribution from the classical theory, so we only have to show continuity. Similarly as before, define

$$
\Phi(\mathbf{y})=\rho_{2}(\mathbf{y})\left\langle u(\mathbf{x}), \rho_{1}(\mathbf{x}) \varphi(\mathbf{x}+\mathbf{y})\right\rangle
$$

which is a function of class $C^{\infty}$ with compact support by Lemma 30 . Here $\rho_{1}$ and $\rho_{2}$ are cutoff functions equal to 1 on $K_{1}$ and $K_{2}$ as given in condition (5). The proof of Lemma 30 gives us

$$
\|\Phi\|_{c^{q, \beta}\left(K_{2}\right)} \lesssim\|\varphi\|_{\mathrm{c}^{r+q+[\alpha+\beta],\{\alpha+\beta\}}\left(K_{1} \times K_{2}\right)}
$$

Therefore, 


$$
\begin{aligned}
|\langle u * v, \varphi\rangle| & =\left|\left\langle v(\mathbf{y}), \rho_{2}(\mathbf{y})\left\langle u(\mathbf{x}), \rho_{1}(\mathbf{x}) \varphi(\mathbf{x}+\mathbf{y})\right\rangle\right\rangle\right| \\
& =|\langle v(\mathbf{y}), \Phi(\mathbf{y})\rangle| \\
& \lesssim\|\Phi\|_{c^{q, \beta}\left(K_{2}\right)} \\
& \lesssim\|\varphi\|_{\mathrm{c}^{r+q+[\alpha+\beta],\{\alpha+\beta\}}\left(K_{1} \times K_{2}\right)},
\end{aligned}
$$

which proves our claim.

Corollary 33 Assume $u \in \mathcal{D}_{(r+\alpha)+}^{\prime}\left(\mathbf{R}^{d}\right)$ and $v \in \mathcal{D}_{q+\beta}^{\prime}\left(\mathbf{R}^{d}\right)$ satisfy the compatibility condition on their supports (5). Then, $u * v \in \mathcal{D}_{(r+q+\alpha+\beta)+}^{\prime}\left(\mathbf{R}^{d}\right)$.

\section{Further extensions}

We see no obstacle in extending the notion of real positive order to currents in the sense of de Rham and distributions on manifolds. For example, the construction could follow the one presented in [9].

Luc Tartar posed to us an interesting question which we were not able to answer so far: What can one say about the order of a fractional derivative of a Radon measure? A standard way to calculate the upper bound on the order of a derivative of a distribution is to use partial integration of its action on test functions. However, partial integration depends on the Leibniz formula, but there is no Leibniz formula for a fractional derivative of a product, so this question is not trivial. We believe that the notion of Hölder distributions might prove suitable to answer this question.

This leads us to a result by Ornstein [21], whose consequence is an answer to a question by Laurent Schwartz: Does there exist a distribution $u \in \mathcal{D}\left(\mathbf{R}^{2}\right)$ such that $\partial_{x} u$ and $\partial_{y} u$ are of order 1 , but $u$ is not of order 0 ? He constructed an example of a distribution in $\mathbf{R}^{2}$ which is not a measure, but whose first-order derivatives were distributions of order 1. Can we use the notion of Hölder distributions to determine the order of $u$ given by Ornstein? Is it possible to give an estimate on the order of all such distributions?

These are closely related to the question of extending two important results in distribution theory. The first one is the structure theorem of distributions which states that any distribution can be locally represented as a finite derivative of a continuous function. Is it possible to have an optimal (in some sense) representation of Hölder distributions using fractional derivatives? The second one is the Schwartz kernel theorem, which states that for every continuous linear map $K: \mathcal{D}(Y) \rightarrow \mathcal{D}^{\prime}(X)$ there exists a unique $k \in \mathcal{D}^{\prime}(X \times Y)$ such that $\langle K v, u\rangle=\langle k, u \otimes v\rangle$. The connection between the order of distributions in the image of $K$ and the order of the distribution $k$ was done in the $\mathrm{PhD}$ thesis of the first author [19] (it is available online). The structure theorem was vital. Is it possible to obtain a similar result in the case when the image of the operator $K$ is a subset of Hölder distributions?

We hope that some of these questions will be resolved soon.

At the end, let us mention an interesting extension communicated to us by an anonymous referee: Instead of using norms of Hölder spaces, one could use norms of more general function spaces. For example, Hölder-Zygmund spaces coincide with Hölder spaces in the case where $\Omega$ is the whole $\mathbf{R}^{d}$ and exponent is a positive non-integer real number, but these spaces do not have $\mathrm{C}_{c}^{\infty}$ as a dense subset since they are not separable (as discussed in Sect. 2). But generally for Besov spaces the approach considered in this article should work. It would be of interest to follow the suggested alternative approach and to investigate the topological properties of the resulting spaces. 
Acknowledgements The idea of extending the notion of the order of distributions from natural numbers to positive reals was suggested to the first author by Luc Tartar during the Generalized Functions 2016 conference in Dubrovnik. The authors are gratefully indebted for his valuable comments and suggestions. The research of the first author was supported in part by the Croatian Science Foundation, Project Number 9780 Weak convergence methods and applications (WeConMApp). A part of the work was performed, while the first author was visiting University Paris-Sud XI under the scholarship of the Government of the French Republic whose support he gratefully acknowledges. A part of the work of the second author was performed while working at the Christian-Albrechts University in Kiel where he was supported by the Deutsche Forschungsgemeinschaft, Project Number 237750060 Fragen der Harmonischen Analysis im Zusammenhang mit Hyperfächen.

\section{References}

1. Adams, R.A., Fournier, J.J.F.: Sobolev Spaces. Academic Press, Cambridge (2003)

2. Alimov, S.A., Ashurov, R.R., Pulatov, A.K.: Multiple Fourier series and Fourier integrals. Commutative harmonic analysis. IV: harmonic analysis in $\mathbf{R}^{n}$. Encycl. Math. Sci. 42, 1-95 (1992)

3. Bari, N.K.: A Treatise on Trigonometric Series. Pergamon Press, Oxford (1964)

4. Beckenstein, E., Narici, L.: Topological Vector Spaces. CRC Press, Boca Raton (2011)

5. Bonahon, F.: Transverse Hölder distributions for geodesic laminations. Topology 36, 103-122 (1997)

6. Bonahon, F.: Geodesic laminations with transverse Hölder distributions. Ann. scient. Éc. Norm. Sup. 30, 205-240 (1997)

7. Bonahon, F.: Geodesic laminations on surfaces. Contemp Math 269, 1-37 (2001)

8. Bonahon, F.: Closed Curves on Surfaces (monograph in preparation)

9. Dieudonné, J.A.: Treatise on Analysis III. Academic Press, Cambridge (1972)

10. Domański, P.: On the separable topological vector spaces. Funct. Approx. Comment. Math. 14, 117-122 (1984)

11. Dubinsky, E.: The Structure of Nuclear Fréchet Spaces. Springer, Berlin (1979)

12. Edwards, R.E.: Functional Analysis: Theory and Applications. Dover Publications, Mineola (1965)

13. Garnir, H.G., De Wilde, M., Schmets, J.: Analyse Fonctionnelle. Birkhäuser Verlag, Basel (1968)

14. Grafakos, L.: Classical Fourier Analysis. Springer, Berlin (2014)

15. Gilbarg, D.: Neil Sidney Trudinger: Elliptic Partial Differential Equations of Second Order. Springer, Berlin (2001)

16. Hörmander, L.: The Analysis of Linear Partial Differential Operators I. Springer, Berlin (2003)

17. Komatsu, H.: Projective and injective limits of weakly compact sequences of locally convex spaces. J. Math. Soc. Jpn. 19, 366-383 (1967)

18. Lohman, R.H.: Wilbur Janes Stiles: on separability in linear topological spaces. Proc. Am. Math. Soc. 42, 236-237 (1974)

19. Mišur, M.: H-distributions and compactness by compensation, Ph.D. thesis, University of Zagreb (2017)

20. Mityagin, B.S.: Approximate dimension and bases in nuclear spaces. Uspekhi Mat. Nauk 16, 63-132 (1961)

21. Ornstein, D.: A non-inequality for differential operators in the $\mathrm{L}^{1}$-norm. Arch. Ration. Mech. Anal. 11, 40-49 (1962)

22. Schaefer, H.H., Wolff, M.P.: Topological Vector Spaces, 2nd edn. Springer, Berlin (1999)

23. Schwartz, L.-M.: Théorie des Distributions. Hermann, Paris (1966)

24. Tartar, L.: An Introduction to Sobolev Spaces and Interpolation Spaces. Springer, Berlin (2007)

25. Trèves, F.: Topological Vector Spaces, Distributions and Kernels. Dover Publications, Mineola (2006)

26. Triebel, H.: Theory of Function Spaces III. Birkhäuser, Basel (2006)

27. Zygmund, A.: Trigonometric Series. Cambridge University Press, Cambridge (2002)

Publisher's Note Springer Nature remains neutral with regard to jurisdictional claims in published maps and institutional affiliations. 